1

\title{
Axon guidance at the midline - a live imaging perspective
}

\author{
Alexandre Dumoulin ${ }^{1) 2)}$, Nikole R. Zuñiga ${ }^{1) 2)}$, Esther T. Stoeckli ${ }^{1)^{2}}$
}

1) Department of Molecular Life Sciences, University of Zurich, Winterthurerstrasse 190, 8057 Zurich, Switzerland

2) Neuroscience Center Zurich, University of Zurich, 8057 Zurich, Switzerland.

Correspondence: Esther T. Stoeckli, e-mail: esther.stoeckli@mls.uzh.ch

Running title: Live imaging of axon guidance.

Key words: axon guidance, midline, spinal cord culture, live imaging, commissural neurons, neural circuit formation, floor plate.

\section{For Development, Techniques and Resources, Research Article}


Summary statement ( 30 words)

Live tracking of single growth cones is more informative about axonal behavior during navigation than inference of behavior from the analyses of snapshots of different growth cones.

\section{ABSTRACT (180 words)}

During neural circuit formation, axons navigate several choice points to reach their final target. At each one of these intermediate targets, growth cones need to switch responsiveness from attraction to repulsion in order to move on. Molecular mechanisms that allow for the precise timing of surface expression of a new set of receptors that support the switch in responsiveness are difficult to study in vivo. Mostly, mechanisms are inferred from the observation of snapshots of many different growth cones analyzed in different preparations of tissue harvested at distinct time points. However, to really understand the behavior of growth cones at choice points, a single growth cone should

42 be followed arriving at and leaving the intermediate target.

43 Here, we describe a spinal cord preparation that allows for live imaging of individual 44 axons during navigation in their intact environment. The possibility to observe single 45 growth cones navigating their intermediate target allows for measuring growth speed, 46 changes in morphology, or aberrant behavior. Moreover, observation of the intermediate 47 target - the floor plate - revealed its active participation and interaction with commissural axons during midline crossing. 


\section{INTRODUCTION}

51 Commissural axons in the developing spinal cord have been used for over two decades

52 to learn fundamental molecular mechanisms of axon guidance (Stoeckli, 2018). The

53 focus was on the dl1 subtype of dorsal commissural interneurons, as their axons have a

54 very stereotypical trajectory at the ventral midline, where they all cross the floor plate

55 (FP), exit it and turn rostrally along the contralateral border. Thus, dl1 commissural

56 neurons offer an easy read-out for deciphering molecular mechanisms of axon guidance

57 at choice points. Since the first application of lipophilic dye tracing in open-book

58 preparations of rat spinal cords that revealed the normal trajectory of these axons 30

59 years ago (Bovolenta and Dodd, 1990), this method continues to be used to assess

60 axon guidance at the midline in mouse and chicken embryos. The comparison between

61 axons in open-book preparations of control and experimentally manipulated spinal

62 cords, dissected at specific time points, offered a solid understanding of molecules

63 involved in axonal midline crossing and subsequent turning in higher vertebrates.

64 However, the information about mechanisms that can be extracted from such

65 experiments is limited, as it is deduced from snapshots of axons taken from different

66 animals. For this reason, we have established a live-imaging approach that allows for

67 visualization of axonal behavior while they are crossing the midline and then turning

68 rostrally. We have chosen the chicken embryo, as it is a very accessible model for

69 studying various developmental processes in intact tissues in vivo and ex vivo (Sanders

70 et al., 2013; Das and Storey, 2014; Boubakar et al., 2017; Li et al., 2019). Thanks to a

71 very stable and reproducible spinal cord culture, we could for the first time characterize

72 the exact timing of midline crossing and the details of rostral turning by dl1 axons in an

73 intact environment in control and experimentally manipulated spinal cords. We could get

74 more insight into growth cone dynamics and morphologies at choice points.

75 Furthermore, our ex vivo method also shed new light on the role of the intermediate

76 target, the FP cells, their dynamics, morphology and interaction with commissural axons

77 during midline crossing. 


\section{Electroporation is an efficient tool to selectively label dl1 neurons}

81 We used unilateral in ovo electroporation of the chicken spinal cord in Hamburger and

82 Hamilton $(\mathrm{HH})$ stage 17-18 embryos to specifically express farnesylated td-Tomato (td-

83 Tomato-F) in Math1-positive dl1 neurons, as well as farnesylated EGFP (EGFP-F) expression in their environment (Fig. 1A,B). One day after electroporation, at HH22, embryos showed expression of td-Tomato-F restricted to dl1 neurons and EGFP-F expression in the entire half of the spinal cord, as expected (Fig. 1C) (Wilson and Stoeckli, 2011). At this stage, most of the Math1-positive dl1 axons approached the FP, but did not yet cross the midline, whereas other more ventral populations of commissural neurons expressing EGFP-F already projected many axons to the contralateral side of the spinal cord (white arrowheads and arrow, respectively, Fig. 1C). For this reason, we chose $\mathrm{HH} 22$ as the optimal stage to start tracing dl1 axons at the

92 midline using live imaging.

\section{Live imaging of dl1 axons at the midline of intact spinal cord}

We extracted the intact spinal cord one day after electroporation (Fig. S1), cultured it with the ventral midline down and imaged it with an inverted spinning disk microscope and a 20x objective (Fig. 1D-F). Math1-positive dl1 axons crossing the FP could be visualized for at least 24 hours (white arrowheads, Fig. 2A; Movies 1, 2). Within this time window many dl1 axons crossed the midline, exited the FP, turned rostrally and formed the contralateral ventral funiculus (white arrows, Fig. 2A). A Math1-positive ipsilateral subpopulation of axons could also be seen in these recordings as previously reported in vivo (Phan et al., 2010) (white asterisk, Fig. 2A). Cultures of intact spinal cords turned out to be a very stable system as the U-shaped morphology of the commissure was preserved over time (Fig. 2B, Movie 2) and all major cell populations were still in place after one day ex vivo (Fig. S2). Furthermore, Sonic hedgehog (Shh) expression was still restricted to the FP and showed the caudal (high) to rostral (low) gradient like in vivo (Fig. S3) (Bourikas et al., 2005), and most importantly, dl1 axons' navigation was identical to the in vivo situation during this time window (Fig. S4). We also compared our ex vivo method with a recently published protocol using open-book 
110 preparations of HH24-26 chicken spinal cords (Pignata et al., 2019) (Fig. 1G-I). In

111 contrast to this protocol, our ex vivo method did not result in overshooting axons, an

112 artefact that was already seen after short times in cultures of open-book preparations,

113 with Math1-positive dl1 commissural axons that crossed the midline but then failed to

114 turn into the longitudinal axis and continued to grow straight into the contralateral side 115 instead (white arrowheads, Fig. 2C,D; Movie 3). Although open-book cultures offer the 116 possibility to follow midline crossing of dl1 axons, the deformation of the FP and 117 commissure in this preparation (Fig. 2D) and the fact that diffusible guidance cues are 118 not well retained in the tissue, most likely lead to these artifacts. These problems were 119 not seen in our cultures of intact spinal cords with meninges attached that prevents 120 diffusion of secreted molecules and preserves gradients (Fig. S3). Therefore, our ex 121 vivo method of culturing intact spinal cords offers a highly stable intact system in which

122 dl1 commissural axons are behaving as expected based on what is known from in vivo 123 studies.

125 Characterization of the timing of midline crossing by dl1 commissural axons

126 The time it takes commissural axons to cross the FP has been estimated but could not 127 be measured exactly (Stoeckli, 2018; Zou, 2012). However, timing is an issue, because 128 axons have to change their responsiveness to FP-derived guidance cues, like the Slits, 129 Shh, or Wnt proteins, by expressing appropriate receptors in a precisely regulated 130 manner (Bourikas et al., 2005; Domanitskaya et al., 2010; Long et al., 2004; 131 Lyuksyutova et al., 2003; Philipp et al., 2012; Wilson and Stoeckli, 2013). With our 132 method, we could track single dl1 commissural axons in the FP at any time point and in 133 different regions of interest (black arrowheads, Fig. 2E). We were therefore able to ask, 134 how long dl1 axons needed for FP crossing and their subsequent rostral turn (Fig. 3A). 135 On average, dl1 commissural axons took $5.6 \pm 1.4 \mathrm{~h}$ to cross the entire FP and $1.4 \pm 1.0 \mathrm{~h}$ 136 to turn and initiate the rostral growth at the FP exit site. Thus, in total, they needed $1376.9 \pm 1.8 \mathrm{~h}$ from entering the FP to the initiation of their rostral growth (mean \pm standard 138 deviation, Fig. 3B and Table S1). There was no significant difference between the 139 average time of crossing the first versus the second half of the FP (Fig. 3B, Table S1). 
140 This was supported by kymographic analysis of a region of interest within the FP from

141 24-hour time-lapse recordings showing similar growth patterns between the first and 142 second half of the FP at the single axon level (Fig. 3C, black arrowheads in Fig. 3D,

143 Movie 4). Although the kymographic analysis was useful to screen for overall growth 144 pattern of single dl1 axons within the FP (Fig. 3E,F), it was not sensitive enough to detect more subtle changes in growth speed. Hence, we used a virtual tracing tool to

146 follow the movement of the leading edge of each growth cone at each time point (Fig. $3 G)$. With this tool we could extract the instantaneous growth speed for each axon. It turned out that the large majority of them had a fluctuating growth pattern with random acceleration-deceleration pulses that could be observed in early as well as late crossing axons (Fig. 3H, Movie 5, Fig. S5). Another interesting observation was made when we compared the times of crossing the FP and the initiation of rostral growth after turning. It seemed that there was a trend towards reduced time of FP crossing in later crossing axons, but this was not significant ( $p=0.0503$; Fig.3l). However, the time dl1 axons took to turn rostrally at the exit site was significantly reduced over time (Fig. 3J). The latter observation suggest that commissural axons that already turned anteriorly at the contralateral FP exit site might help the following ones to turn more rapidly. Our method offers new opportunities for further investigations of possible collaborations between axons at choice points.

\section{Characterization of the dl1 growth cone morphology at choice points}

Another aspect that we considered was growth cone morphology. The growth cone plays a central role in axon guidance, as it explores the environment for guidance cues and translates this information into the directionality of growth (Stoeckli, 2018; de Ramon Francàs et al., 2017). We observed that dl1 growth cones in the FP appeared to have a thin and elongated shape in the direction of growth. At the FP exit site, they transiently enlarged (arrowheads, Fig. 4D, Movie 2). We measured the average growth cone area in each segment of interest and confirmed that growth cones at the exit site of the FP were indeed significantly larger than the ones within the FP or after the turn 
170 between the first and second half of the FP (Fig. 4B, Table S1). The changes of growth

171 cone shape were in line with previous reports on chicken and rat commissural axons in

172 vivo (Bovolenta and Dodd, 1990; Yaginuma et al., 1991) and our data on Math1-positive

173 axons in vivo (Fig. 4C, Table S1, Fig. S4B). The possibility to follow individual axons

174 over time allowed us to make novel observations of their behavior at the FP exit site.

175 The growth cones very often extended long filopodia in both rostral and caudal direction

176 just before turning (Fig. 4D, Movies 6,7). Some of the growth cones even appeared to

177 transiently split just before turning rostrally, similar to dorsal root ganglia central

178 afferents in the mouse dorsal root entry zone before bifurcating (Dumoulin et al., 2018)

179 (Movie 8). All these features are present in vivo, as similar growth cone morphologies

180 were found in fixed HH24-25 spinal cords (Fig. 4E). Thus, ex vivo live imaging of

181 cultured intact spinal cords using low magnification time-lapse microscopy offers the

182 opportunity to detect morphological changes of growth cones at choice points and is

183 ideal for the analysis of many aspects of midline crossing. However, the limited resolution especially in 3D might preclude the detection of more subtle changes in morphology of growth cones while crossing the midline.

Higher magnification analysis of commissural axons crossing the midline revealed dorso-ventral activities of their growth cone

For this reason, we repeated time-lapse recordings of cultured intact spinal cords using a higher magnification objective and advanced 3D deconvolution technology. This allowed following Math1::tdTomato-F-positive dl1 axons over time while entering, crossing and exiting the FP (Movie 9). We observed that dl1 growth cones crossing the FP were bulkier in the dorso-ventral axis (white arrowheads, Fig. 5A, Movie 10) and were particularly dynamic in this axis showing rapid extension of filopodial protrusions (white arrows in Fig. 5A and Movie 10). This aspect of dl1 growth cone behavior in the FP was supported by immunostaining of $\mathrm{HH} 22-24.5$ whole-mount spinal cords or cryosections (arrowheads, Fig. 5B-D, Movie 11,12). In line with their dorso-ventral protrusion (up to $\sim 13 \mu \mathrm{m}$ ) into the FP while crossing it (black arrow, Fig. 5E, black 
200 arrowheads, Movie 13). Protrusions entering the FP could also be detected for dl1

201 growth cones in vivo as revealed by immunostaining of HH22-24.5 whole-mount spinal

202 cords or cryosections (white arrows, Fig. 5F,G). Next, we also had a closer look at the

203 FP exit site, where growth cones need to read longitudinal gradients to initiate the 204 rostral turn after exiting the FP (Pignata et al., 2019; Stoeckli, 2018). Intriguingly, we 205 detected that just before exiting the FP, dl1 growth cones very often sent a long 206 protrusion into the FP (arrow, Fig. 5H, Movie 14). Live imaging clearly revealed that the 207 activity and orientation of growth cones switched by about $90^{\circ}$ after exiting the FP, as 208 they flattened in the dorso-ventral axis and enlarged in the longitudinal axis ex vivo and 209 in vivo (Fig. 5I,J, Movie 15). Another unexpected observation we made was that some 210 dl1 growth cones transiently split while crossing the FP (asterisks in Movie 13). The 211 splitting created two more or less equal branches (black arrows, Fig. 5K, Movie 16), but 212 only one persisted and grew straight to the contralateral side, while the other one was 213 retracted (black asterisks, Fig. 5K, Movie 16). Also this behavior was supported by 214 snapshots from in vivo behavior of dl1 growth cones (arrows, Fig. 5L). Taken together, 215 ex vivo live imaging combined with high magnification analysis of growth cone dynamics 216 allowed us to characterize the behavior dl1 growth cones at choice points in more 217 detail.

Live imaging unraveled the dynamics and morphologies of floor-plate cells during midline crossing

221 The orientation of dl1 growth cones as well as their behavior during FP crossing 222 suggested that they have to squeeze their way between the basal feet of FP cells which 223 are attached to the basal lamina (Yaginuma et al., 1991; Yoshioka and Tanaka, 1989). 224 Moreover, very little was known about the morphology of FP cells during axonal midline 225 crossing and their potential active contribution in this process has never been 226 addressed (Campbell and Peterson, 1993; Yaginuma et al., 1991; Yoshioka and 227 Tanaka, 1989). Therefore, we examined the behavior and morphology of FP cells 228 during midline crossing in our ex vivo system. We electroporated spinal cords at $\mathrm{HH} 17-$ 22918 after injection of a plasmid encoding EGFP-F under the FP-specific Hoxa1 enhancer 
230 for expression of the membrane-bound fluorescent protein in FP cells (Li and Lufkin,

231 2000; Wilson and Stoeckli, 2011; Zisman et al., 2007) (Fig. 6A-D). With this we were

232 able to see Hoxa1::EGFP-F-positive bulky FP basal feet in the commissure in vivo

233 (white arrowheads, Fig. 6C) as well as their thin morphology and orientation (white

234 arrows) that seemed to be tightly aligned with dl1 growth cones crossing the midline

235 (white arrowheads, Fig. 6E). The morphology of medial FP basal feet with little

236 extension in the rostro-caudal axis but enlarged in the dorso-ventral axis could be

237 observed in real time using our ex vivo culture technique (Fig. 6F, Movie 17).

238 Interestingly, we could observe dynamic protrusions sprouting from the basal feet in

239 direction of axonal growth in the commissure (black arrows, Fig. 6F, Movie 17). Similar

240 observations were made for basal feet of lateral FP cells (black arrow, Fig. 6G). They

241 showed a very high activity with very dynamic protrusions towards the FP entry zone

242 (black arrows, Fig. 6G, Movie 18). Importantly, we could observe a similar morphology

243 of medial FP basal feet at the single-cell level in vivo (arrowheads, Fig. 6H, Movie 19).

244 The dl1 commissural axons had the same orientation as the FP basal feet and seemed

245 to grow in between these feet and interact with them in vivo (Movie 19 and 20).

246 Moreover, we could observe similar protrusions coming either from lateral FP basal feet

247 (white arrows) going towards pre-crossing dl1 axons arriving at the FP (white

248 arrowheads, Fig. 6I), or from medial FP basal feet extending parallel to axons in the

249 commissure (white arrows, Fig. 6J and Movie 21). The tight interaction between dl1

250 axons and FP basal feet during midline crossing was unexpected (Movie 21). The

251 observation that protrusions from lateral FP basal feet (white arrowhead) were

252 extending and contacting dl1 growth cones before they entered the FP (white arrows)

253 suggested a much more active role of FP cells than anticipated (Fig. 6K, Movie 22).

254 Last but not least, we could confirm that FP basal feet structures (yellow arrow) were

255 present in between transiently splitting dl1 growth cones (white arrowheads and arrows)

256 in the commissure (Fig. 6L, Movie 23). Taken together, these data showed for the very

257 first time the detailed morphology of single FP cells, their dynamics and tight

258 interactions with axons during midline crossing, and they emphasized the probable

259 active role of FP cells in initiating contacts with growth cones before and during midline

260 crossing. 
Trajectory and behavior of dl1 axons can be visualized in real time at choice points

264 Our ex vivo culture method not only offers great opportunities to characterize behavior of growth cones and FP cells in a preserved system, but it also opens new possibilities for tracking axonal behavior after specific perturbations of either the neurons or their environment. As example, the Wnt receptor Fzd3 (Frizzled-3) was specifically downregulated in Math1-positive dl1 neurons (Alther et al., 2016; Wilson and Stoeckli, 2011) (Fig. 7A). Fzd3 is required for the rostral turn of post-crossing commissural axons at the contralateral FP border in vivo (Alther et al., 2016; Lyuksyutova et al., 2003). We used our ex vivo culture system to visualize Math1::EGFP-F-positive dl1 axons expressing a microRNA for Fzd3 (miFzd3, Fig. 7B,C). This allowed us to follow in real time how dl1 growth cones were turning caudally instead of rostrally at the contralateral FP border (black arrowheads, Fig. 7B; Movie 24 and 25). Interestingly, many axons were found to turn erroneously in caudal direction at the same position, suggesting that axons were influenced by close contact with other axons (black asterisk and black arrows in Movie 24). In addition, some axons were found stalling at the FP exit site without initiating any turn (black arrowhead, Fig. 7C, Movie 25). Nonetheless, the growth cones remained highly dynamic. The changes in morphology were accompanied with transient retraction and re-extension but without a clear change in directionality. Importantly, expressing a control microRNA (mi2Luc) did not impact the guidance of dl1 axons at the contralateral FP border (Fig. 7D, Movie 26). Taken together, our method can be used to study the behavior of axons after perturbation of candidate genes specifically in the neurons or their target in real time.

\section{DISCUSSION}

287 The possibility to follow one axon over time, rather than deducing behavior from 288 snapshots of different axons, allowed us to extract detailed information on the timing of 289 midline crossing and the tight interaction between FP cells and the growth cones in a 
290 higher vertebrate model (Figure 8). Our ex vivo system offers significant improvement 291 over existing open-book culture systems which lead to obvious guidance artefacts at the 292 contralateral FP border (Figure 2) (Pignata et al., 2019). Our ex vivo culture system is 293 highly reproducible and generates a manageable amount of data compared to live 294 imaging using light sheet-based microscopy, for example (Liu et al., 2018).

295 Our comparative analyses demonstrate that midline crossing of dl1 axons in our ex vivo 296 system was very similar to what happens in vivo (Figure 2,4,5 and S4). Therefore, our ex vivo system can be used to monitor and assess axonal behavior at choice points. We could detect that dl1 growth cones took on average 5.6 hours to cross the entire FP and that they did so in a pulsed manner (black arrowheads, Figure 8). We could also measure that they needed on average 1.4 hours to initiate their rostral growth, and that the first axons exiting the FP took longer than the followers (Figure 8A). In total they needed almost 7 hours from entering the FP to making the decision to turn rostrally (blue arrowhead, Figure 8A). This is enough time for growth cones to change their responsiveness to specific guidance cues for crossing and exiting the FP as well as for turning rostrally due to changes in receptor expression regulated at the posttranslational, translational and even transcriptional level (Nawabi et al., 2010; Philipp et al., 2012; Pignata et al., 2019; Preitner et al., 2016; Stoeckli, 2018; Wilson and Stoeckli, 2013).

The growth cone is the decision center where axon guidance instructions are transduced to the cytoskeleton (Vitriol and Zheng, 2012). With our newly developed ex vivo system, dynamic changes in dl1 commissural growth cone morphology and 312 behavior at the midline can be observed in real time. Growth cones were thin and 313 elongated in the FP with their major extension in the dorso-ventral axis (black 314 arrowheads, Figure 8A,C). At the FP exit site, they showed a $90^{\circ}$ rotation to be enlarged and active in the longitudinal axis (green arrowhead and black arrow, Figure 8A, black 316 arrowhead, Figure 8D). The fact that dl1 growth cones sent a long filopodium into the 317 FP, towards the FP cell soma area, while crossing it and just before exiting it, suggests 318 that they might need to read signals from this area in order to move on and exit the FP 319 (orange arrows in Figure 8C and D). The extension of long filopodia just before FP exit 
320 and rostral turning suggests that actin polymerization might be required to sense

321 repulsive cues - for instance SlitN and Shh, respectively - and transduce the signal into

322 the growth cone, as suggested for Slit-induced growth cone collapse in vitro (McConnell

323 et al., 2016). Further investigations using our ex vivo culture system will be required to

324 understand the role of cytoskeletal dynamics in axonal navigation of the intermediate

325 target.

326 Our method also suggested a probable active contribution of FP cells to axon guidance, 327 as we found the cells of the intermediate target to by very dynamic and to extend 328 protrusions in directions of the arriving axons, or to actively engage with axons in the FP. Thus, it seems that the intermediate target is much more than a passive by-stander and provider of attractive and repulsive axon guidance and cell adhesion molecules. We characterized the FP cell morphologies in detail in the medial as well as the lateral FP. Basal feet appeared to be enlarged and oriented parallel to commissural growth cones

333 (Figure 8C). The lateral FP basal feet sent protrusions (black arrows) towards dl1 334 growth cones approaching the FP and eventually interacted with them (black 335 arrowhead, Figure 8B). This intriguing observation led us to speculate whether these 336 protrusions might be cytonemes. Cytonemes are long protrusions known to spread and 337 deliver morphogens, such as Wnts and Shh, to neighboring or more distant cells 338 (González-Méndez et al., 2019; Sanders et al., 2013; Stanganello and Scholpp, 2016). 339 Given the fact that Shh is involved in guiding pre-crossing commissural axons towards the FP and that Shh and Wnts are both involved in guiding post-crossing axons towards

341 the brain at the contralateral FP border, it is tempting to speculate that these protrusions 342 might deliver such signals to the growth cones at choice points (Avilés et al., 2013). 343 Moreover, we could appreciate how much the axons and their growth cones were 344 intermingled within the medial FP basal feet which also formed long dynamic 345 protrusions within the commissure (black arrowheads, Figure 8C). In sum, the 346 combination of our live imaging approach with a FP-specific marker will give the 347 opportunity to further characterize the behavior of intermediate target cells with regard 348 to axon guidance at choice points. 
Ultimately, our method will be useful to get more insights into molecular mechanisms of axon guidance at a choice point, when combined with in ovo RNAi for specific gene knockdowns either in the neurons or in their environment, as exemplified with Fzd3 knockdown experiments (Figure 7) (Andermatt et al., 2014; Pekarik et al., 2003). Similarly, pharmacological blockers will permit to screen for components required downstream of growth cone receptors to transduce guidance signals. Usually such experiments are conducted in vitro with cultured neurons growing axons in a very artificial environment. Thus, our method offers the advantages of an in vitro experiment in an intact complex 'in vivo-like' environment. The use of specific reporters will also allow for the assessment of dynamic changes of second messengers or the actin cytoskeleton in growth cones for example (Nicol et al., 2011; Nichols and Smith, 2019). Moreover, the use of other sets of enhancers and promoters might offer the possibility to study the dynamics of midline crossing in other subtypes of commissural neurons in the spinal cord and in the brain (Hadas et al., 2014; Kohl et al., 2012).

\section{MATERIALS AND METHODS}

\section{In ovo electroporation}

Plasmids encoding farnesylated td-Tomato under control of the Math1 enhancer and the $\beta$-globin promoter for dl1 neuron-specific expression (Math1::tdTomato- $F, 700 \mathrm{ng} / \mathrm{\mu l}$ ) and farnesylated EGFP under control of the $\beta$-actin promoter ( $\beta$-actin::EGFP-F, 30 $\mathrm{ng} / \mathrm{\mu l}$ ) were co-injected into the central canal of the chicken neural tube in ovo at $\mathrm{HH} 17$ 18 (Hamburger and Hamilton, 1951) and unilaterally electroporated, using a BTX ECM830 square-wave electroporator (five pulses at $25 \mathrm{~V}$ with $50 \mathrm{~ms}$ duration each), as previously described (Wilson and Stoeckli, 2012) (Fig. 1A,B). A final concentration of $0.1 \%(\mathrm{vol} / \mathrm{vol})$ of Fast Green was added to the plasmid mix to trace injection site and volume of the plasmid mix. After electroporation, embryos were covered with sterile PBS and eggs were sealed with tape and incubated at $39^{\circ} \mathrm{C}$ for $26-30$ hours, until embryos reached stage $\mathrm{HH} 22$, or for 36-46 hours, until embryos reached $\mathrm{HH} 24$. For the FP study EGFP-F was expressed from a plasmid with the Hoxa1 enhancer and the $\beta$ globin minimal promoter (Wilson and Stoeckli, 2011) (Hoxa1::EGFP-F, $1000 \mathrm{ng} / \mu \mathrm{l}$ ) and 
co-injected with the Math1::tdTomato-F plasmid $(700 \mathrm{ng} / \mu \mathrm{l})$ and unilaterally electroporated as above, or bilaterally electroporated (3 pulses in each direction at $25 \mathrm{~V}$ with $50 \mathrm{~ms}$ duration each). For knockdown of Fzd3 (or luciferase as control) in dl1 neurons (Math1 enhancer) plasmids previously published (Math1::EGFP-F; miFzd3 and Math1::EGFP-F; mi2Luc, $700 \mathrm{ng} / \mu \mathrm{l})$ were co-injected with the $\beta$-actin::mRFP plasmid (30 ng/ $\mu \mathrm{l})$ and unilaterally electroporated at $\mathrm{HH} 16$ for more efficient knockdown (Alther et al., 2016).

\section{Dissection of intact spinal cords}

Intact spinal cords were dissected from HH22 embryos in ice-cold, sterile PBS (Gibco) in a silicon-coated Petri dish with sterile instruments. Embryos were pinned down with their dorsal side down with thin needles (insect pins). Here, special care was taken not to damage or detach meninges surrounding the spinal cord by avoiding too much rostro-caudal and lateral tension. Internal organs and ventral vertebrae were removed to access the spinal cord. Ventral roots were cut off and the spinal cord was carefully extracted from the embryo with forceps, avoiding any excessive bending. Note that dorsal root ganglia were not cut off and all were still attached to the spinal cord. The ventral and dorsal midline were kept intact throughout dissection. Finally, remaining dorsal tissues were discarded. See Fig. S1 for a detailed step-by-step protocol to successfully dissect intact $\mathrm{HH} 22$ spinal cords. Note that this procedure can also be applied to older embryos (at least HH24-25). Once intact spinal cords were dissected and cleaned from any remaining dorsal tissues they were embedded with the ventral side down in a warm $\left(39^{\circ} \mathrm{C}\right) 100-\mu$ l drop of $0.5 \%$ low-melting agarose (FMC, Fig. 1D,E, Fig. S1G) containing a 6:7 ratio of spinal cord medium [MEM with Glutamax (Gibco) supplemented with $4 \mathrm{mg} / \mathrm{ml}$ Albumax (Gibco), $1 \mathrm{mM}$ pyruvate (Sigma), 100 Units/ml Penicillin and $100 \mu \mathrm{g} / \mathrm{ml}$ Streptomycin (Gibco)] in a 35-mm Ibidi $\mu$-Dish with glass bottom (Ibidi, \#81158). Note that the spinal cord should be as straight as possible with the dorso-ventral axis perpendicular to the glass bottom, as any pronounced curvature or tilting of the midline would induce axon guidance artefacts or death of the axons, respectively. Once the agarose solidified (around $5 \mathrm{~min}$ at room temperature), $200 \mu \mathrm{l}$ of 
spinal cord medium were added to the drop and the culture could be started. A 12-mm flexiPERM conA ring (Sarstedt) was placed in the center of the culture dish before the agarose drop was added (the drop should not touch the ring). Hence, the medium added to the drop of low-melting agarose could touch the ring all around and therefore stabilize the position of the agarose drop and avoid any movement of the spinal cord during recordings thanks to surface tension (Fig. S1G).

\section{Dissection of open-books}

Open-book preparations of spinal cords were dissected from $\mathrm{HH} 24$ embryos as previously described in a video protocol for HH25-26 embryos (Wilson and Stoeckli, 2012). The first steps were identical to the protocol for intact spinal cord dissection given above (steps A,B in Fig. S1). Starting there, the tension along the rostro-caudal axis was increased using the upper and lower needles and meninges were removed with a blade made of fire-polished tungsten wire. Spinal cords were cut transversally at the wing and leg levels and carefully extracted from the embryo with forceps. At this point, the dorsal midline spontaneously opened. Open-book preparations of spinal cords were then plated with the apical side down (Fig. $1 \mathrm{G}, \mathrm{H}$ ) in the center of a $35-\mathrm{mm}$ Ibidi $\mu$ Dish with glass bottom (Ibidi, \#81158), pre-coated with $20 \mu \mathrm{g} / \mathrm{ml}$ poly-L-lysine (Sigma). A homemade, harp-like holder made out of a Teflon ring and thin nylon strings was used to keep the spinal cord in place (Fig. 1G). Note that the strings were barely touching the open-books but stabilized the flat position of the spinal cord. Then, $100 \mu \mathrm{l}$ of $0.5 \%$ low-melting agarose (FMC) containing a 6:7 ratio of spinal cord medium were added on top of the spinal cord. Once the agarose solidified (around $5 \mathrm{~min}$ at room temperature), $200 \mu \mathrm{l}$ of spinal cord medium were added to the agarose drop and the culture could be started.

\section{Live imaging}

Live imaging recordings were carried out with an Olympus IX83 inverted microscope equipped with a spinning disk unit (CSU-X1 10,000 rpm, Yokogawa). Cultured spinal 
438 cords were kept at $37^{\circ} \mathrm{C}$ with $5 \% \mathrm{CO}_{2}$ and $95 \%$ air in a PeCon cell vivo chamber

439 (PeCon). Temperature and $\mathrm{CO}_{2}$-levels were controlled by the cell vivo temperature controller and the $\mathrm{CO}_{2}$ controller units (PeCon). Spinal cords were incubated for at least 30 min before imaging was started. We acquired 18-40 planes (1.5 $\mu$ m spacing) of 2x2

442 binned z-stack images every 15 min for 24 hours with a 20x air objective (UPLSAPO $44320 x / 0.75$, Olympus) and an Orca-Flash 4.0 camera (Hamamatsu) with the Olympus 444 CellSens Dimension 2.2 software. We performed most of our recordings in the lumbar 445 level of the spinal cord and always took 3 channels of interest: emission at $488 \mathrm{~nm}$ and $446561 \mathrm{~nm}$, as well as brightfield. Recordings of Fzd3 or luciferase knockdown axons were 447 performed at the thoracic level. For higher magnification recordings, a 40x silicone oil 448 objective was used (UPLSAPO S 40x/1.25, Olympus) with same acquisition settings as above and images taken every 5-15 $\mathrm{min}$.

\section{Data processing and virtual tracing}

452 Z-stacks and maximum projections of Z-stack movies were evaluated and processed 453 using Fiji/lmageJ (Schindelin et al., 2012). The MtrackJ plugin (Meijering et al., 2012) 454 was used to virtually trace single Math1-positive dl1 commissural axons crossing the 455 FP. This helped to keep track of which axons had already been quantified. The leading edge (and not filopodia) of growth cones was always selected for each time point. At the exit site, as growth cones very often slightly changed their directionality and drastically change their shape before turning, the central domain of the growth cone was selected with the tracing tool. Only axons that entered, crossed and exited the FP during the 24hour imaging period were traced and quantified. Overlays of labeled axons with EGFP-F and brightfield channels were used to assess the FP boundaries and midline localization. The virtual tracing tool was also used to extract the instantaneous growth speed for each single axon. Note that the montage of dl1 commissural axons shown in Movie 2 was generated from z-stacks that were 2D deconvolved (nearest neighbor) 465 using the Olympus CellSens Dimension 2.2 software and assembled with Fiji/lmageJ. 466 All data acquired with higher magnification (40x silicone oil objective) were 3D 467 deconvolved using constrained iterative deconvolution of the Olympus CellSens 
Dimension 2.2 software (5 iterations with adaptive PSF and background removal, Olympus). Maximum projections of live images containing Hoxa1::EGFP-F-positive cells (channel) were corrected for photo bleaching in Fiji/lmageJ.

\section{Temporal-color projections and kymographic analysis}

Temporal-color projections were generated using Fiji/lmageJ. Kymograph analysis of axons crossing or exiting the FP as previously described (Medioni et al., 2015) using a region of interest $(\mathrm{ROI})$ selection, the re-slice function and the z-projection of the resliced results in Fiji/lmageJ, which allowed following pixel movements within the horizontal axis. The ROI in the FP was selected as a $103 \times 51 \mu \mathrm{m}^{2}$ (Fig. 3C,D) or $103 \times 27$ $\mu \mathrm{m}^{2}$ (Fig. 3E,F) rectangle.

\section{Immunohistochemistry}

Spinal cords dissected from HH22 embryos or intact spinal cords that were cultured for one day ex vivo were fixed one hour at room temperature with $4 \%$ paraformaldehyde in PBS, washed 3 times for 5 min each with PBS and cryopreserved for at least $24 \mathrm{~h}$ at $4^{\circ} \mathrm{C}$ in $25 \%$ sucrose in PBS. After mounting in O.C.T. compound (Tissue-Tek) and freezing the spinal cords, $25-\mu \mathrm{m}$ thick cryosections were collected using a cryostat. The next day, sections were blocked and permeabilized $1 \mathrm{~h}$ at room temperature with $5 \%$ FCS in $0.1 \%$ Triton X-100 in PBS (blocking buffer). Primary antibodies were diluted in blocking buffer and added to sections overnight at $4^{\circ} \mathrm{C}$ (1:400 for goat-anti-GFP-FITC, Rockland; 1:2,500 for rabbit-anti-RFP, antibodies-online; supernatants of monoclonal antibodies obtained from DSHB: anti-Lhx2 (clone 1C11), anti-islet-1, (clone 40.2D6), anti-Nkx2.2 (clone $74.5 \mathrm{~A} 5$ ), anti-Hnf3 $\beta$ (clone $4 \mathrm{C} 7$ ); $3.1 \mu \mathrm{g} / \mathrm{ml}$ of mouse-anti-Shh, clone $5 \mathrm{E} 1$ ). The next day, sections were washed 3 times for 15 min each at room temperature with $0.1 \%$ Triton-X100 in PBS. Primary antibodies (except anti-GFP-FITC) were detected with a 2hour incubation in adequate secondary antibodies diluted in blocking buffer (1:1,000 for donkey-anti-mouse-IgG-Cy5 or donkey-anti-rabbit-lgG-Cy3, Jackson ImmunoResearch). Finally, nuclei were counterstained for $10 \mathrm{~min}$ at room temperature 
497

498

499

500

501

502

503

504

505

506

507

508

509

510

511

512

513

514

515

516

517

518

519

520

521

522

523

524

525

526

with $2.5 \mu \mathrm{g} / \mathrm{ml}$ of Hoechst diluted in 0.1 Triton X-100 in PBS (Invitrogen), washed 3 times for 10 min each with $0.1 \%$ Triton X-100 in PBS and 2 times for 5 min each with PBS before mounting the slides in Mowiol/DABCO. Images were taken with an Olympus IX83 inverted microscope equipped with a spinning disk unit (CSU-X1 10,000 rpm, Yokogawa), a 20x air objective (UPLSAPO 20x/0.8, Olympus) or a $40 x$ silicon oil objective (UPLSAPO S 40x/1.25, Olympus), and an Orca-Flash 4.0 camera (Hamamatsu) with the Olympus CellSens Dimension 2.2 software, or with an Olympus BX61 upright microscope and a 10x air objective (UPLFL PH 10x/0.30, Olympus) or 40x water objective (UAPO W/340 40x/1.15, Olympus) and an Orca- $R^{2}$ camera (Hamamatsu) with the Olympus CellSens Dimension 2.2 software.

\section{Whole-mount immunostaining}

Intact spinal cords dissected from HH24-25 embryos were fixed for $1 \mathrm{~h}$ at room temperature in 4\% paraformaldehyde in PBS and washed 3 times 10 min each with PBS. Spinal cords were permeabilized and incubated for $1 \mathrm{~h}$ at room temperature with $5 \%$ FCS in $0.1 \%$ Triton X-100 in PBS (blocking buffer). Primary antibodies were diluted in blocking buffer and added to spinal cords for incubation overnight a $4^{\circ} \mathrm{C}(1: 800$ of goat-anti-GFP-FITC, Rockland; 1:5,000 of rabbit-anti-RFP, antibodies-online). The next day, sections were washed 3 times 30 min each at room temperature with $0.1 \%$ TritonX100 in PBS. The primary antibody against RFP was detected with a 2-hour incubation in diluted donkey-anti-rabbit-IgG-Cy3 antibody in blocking buffer $(1: 1,000$, Jackson ImmunoResearch). Finally, nuclei were counterstained for $30 \mathrm{~min}$ at room temperature with $2.5 \mu \mathrm{g} / \mathrm{ml}$ of Hoechst diluted in 0.1 Triton X-100 in PBS (Invitrogen). Samples were washed 3 times 30 min each with $0.1 \%$ Triton X-100 in PBS and 2 times 15 min each in PBS. Stained spinal cords where mounted in $100 \mu \mathrm{l}$ of $0.5 \%$ low-melting agarose in PBS with the ventral midline pointing down on a $35-\mathrm{mm}$ lbidi $\mu$-Dish with glass bottom (Ibidi, \#81158), similarly as described above. This allowed accessing the commissure of fixed in vivo samples and visualization of dl1 axons and their growth cone with an Olympus IX83 inverted microscope equipped with a spinning disk unit (CSU-X1 10,000 rpm, Yokogawa). Pictures were taken with a $4 x$ air objective (UPLFLN PH 4x/0.13, 
527 Olympus), a 20x air objective (UPLSAPO 20x/0.75, Olympus) ) or a $40 x$ silicon oil

528 objective (UPLSAPO S 40x/1.25, Olympus) and an Orca-Flash 4.0 camera

529 (Hamamatsu) with the Olympus CellSens Dimension 2.2 software. Note that the same

530 mounting and microscopy procedure was applied to HH23-25 intact spinal cords that

531 were not stained and were used for the quantification of average growth cone areas in

532 vivo (shown in Fig. 4C).

533

534 Statistics and Figures assembly

535 Statistical analyses were carried out with GraphPad Prism 7.02 software. All data were 536 assessed for normality (normal distribution) using the D'Agostino and Pearson omnibus 537 K2 normality test and visual assessment of the normal quantile-quantile plot before 538 choosing an appropriate (parametric or non-parametric) statistical test. $P$ values of the 539 simple linear regression shown in Fig. 3l,J demonstrate whether the slope is 540 significantly different to zero and the dashed lines represent the $95 \%$ confidence 541 intervals. Figures were assembled using Corel Draw 2017.

Acknowledgements

544 We thank Dr. Beat Kunz for excellent technical assistance.

546 Competing interests

547 The authors declare no competing interests.

\section{Author contributions}

$550 \mathrm{AD}$, designed experiments, performed experiments, evaluated data and contributed to 551 the writing of the manuscript, NRZ, generated the Hoxa1 plasmid, ETS, designed 552 experiments, supervised the project and contributed to the writing of the manuscript. 


\section{Funding}

This project was supported by the Swiss National Science Foundation.

References

Alther, T. A., Domanitskaya, E. and Stoeckli, E. T. (2016). Calsyntenin 1-mediated trafficking of axon guidance receptors regulates the switch in axonal responsiveness at a choice point. Development. 143, 994-1004.

Andermatt, I., Wilson, N. and Stoeckli, E. T. (2014). In ovo electroporation of miRNAbased-plasmids to investigate gene function in the developing neural tube. Methods Mol. Biol. 1101, 353-368.

Avilés, E. C., Wilson, N. H. and Stoeckli, E. T. (2013). Sonic hedgehog and Wnt: Antagonists in morphogenesis but collaborators in axon guidance. Front. Cell. Neurosci. 7, 86. doi:10.3389/fncel.2013.00086

Boubakar, L., Falk, J., Ducuing, H., Thoinet, K., Reynaud, F., Derrington, E. and Castellani, V. (2017). Molecular Memory of Morphologies by Septins during Neuron Generation Allows Early Polarity Inheritance. Neuron 95, 834-851.e5.

Bourikas, D., Pekarik, V., Baeriswyl, T., Grunditz, Å., Sadhu, R., Nardó, M. and Stoeckli, E. T. (2005). Sonic hedgehod guides commissural axons along the longitudinal axis of the spinal cord. Nat. Neurosci. 8, 297-304.

Bovolenta, P. and Dodd, J. (1990). Guidance of commissural growth cones at the floor plate in embryonic rat spinal cord. Development 109, 435-447.

Campbell, R. M. and Peterson, A. C. (1993). Expression of a lacZ transgene reveals floor plate cell morphology and macromolecular transfer to commissural axons. Development 119, 1217-1228.

Das, R. M. and Storey, K. G. (2014). Apical abscission alters cell polarity and dismantles the primary cilium during neurogenesis. Science 343, 200-204.

de Ramon Francàs, G., Zuñiga, N. R. and Stoeckli, E. T. (2017). The spinal cord shows the way - How axons navigate intermediate targets. Dev. Biol. 432, 43-52.

Domanitskaya, E., Wacker, A., Mauti, O., Baeriswyl, T., Esteve, P., Bovolenta, P. and Stoeckli, E. T. (2010). Sonic hedgehog guides post-crossing commissural axons both directly and indirectly by regulating Wnt activity. J. Neurosci. 30, 11167-11176.

Dumoulin, A., Dagane, A., Dittmar, G. and Rathjen, F. G. (2018). S-palmitoylation Is Required for the Control of Growth Cone Morphology of DRG Neurons by CNP. Induced cGMP Signaling. Front. Mol. Neurosci. 11:345 doi: 10.3389/fnmol.2018.00345. 
González-Méndez, L., Gradilla, A. C. and Guerrero, I. (2019). The cytoneme connection: Direct long-distance signal transfer during development. Development. 146: $\operatorname{dev} 174607$.

Hadas, Y., Etlin, A., Falk, H., Avraham, O., Kobiler, O., Panet, A., Lev-Tov, A. and Klar, A. (2014). A "tool box" for deciphering neuronal circuits in the developing chick spinal cord. Nucleic Acids Res. 42, e148. doi:10.1093/nar/gku750.

Hamburger, V., and Hamilton, H.L. (1951). A series of normal stages in the development of the chick embryo. J. Morphol. 88, 49-92. Kohl, A., Hadas, Y., Klar, A. and Sela-Donenfeld, D. (2012). Axonal patterns and targets of dA1 interneurons in the chick hindbrain. J. Neurosci. 32, 5757-5771.

Li, X. and Lufkin, T. (2000). Cre recombinase expression in the floorplate, notochord and gut epithelium in transgenic embryos driven by the Hoxa-1 enhancer III. Genesis 26, 121-122.

Li, Y., Vieceli, F. M., Gonzalez, W. G., Li, A., Tang, W., Lois, C. and Bronner, M. E. (2019). In Vivo Quantitative Imaging Provides Insights into Trunk Neural Crest Migration. Cell Rep. 26, 1489-1500.e3.

Liu, T. L., Upadhyayula, S., Milkie, D. E., Singh, V., Wang, K., Swinburne, I. A., Mosaliganti, K. R., Collins, Z. M., Hiscock, T. W., Shea, J., et al. (2018). Observing the cell in its native state: Imaging subcellular dynamics in multicellular organisms. Science 360, eaaq1392.

Long, H., Sabatier, C., Ma, L., Plump, A., Yuan, W., Ornitz, D. M., Tamada, A., Murakami, F., Goodman, C. S. and Tessier-Lavigne, M. (2004). Conserved roles for Slit and Robo proteins in midline commissural axon guidance. Neuron 42, 213223.

Lyuksyutova, A. I., Lu, C. C., Milanesio, N., King, L. A., Guo, N., Wang, Y., Nathans, J., Tessier-Lavigne, M. and Zou, Y. (2003). Anterior-Posterior Guidance of Commissural Axons by Wnt-Frizzled Signaling. Science 302, 1984-1988.

McConnell, R. E., van Veen, J. E., Vidaki, M., Kwiatkowski, A. V., Meyer, A. S. and Gertler, F. B. (2016). A requirement for filopodia extension toward Slit during Robo-mediated axon repulsion. J. Cell Biol. 213, 261-274.

Medioni, C., Ephrussi, A. and Besse, F. (2015). Live imaging of axonal transport in Drosophila pupal brain explants. Nat. Protoc. 10, 574-584.

Meijering, E., Dzyubachyk, O. and Smal, I. (2012). Methods for cell and particle tracking. In Methods in Enzymology, 504, pp. 183-200.

Nawabi, H., Briançon-Marjollet, A., Clark, C., Sanyas, I., Takamatsu, H., Okuno, T., Kumanogoh, A., Bozon, M., Takeshima, K., Yoshida, Y., et al. (2010). A midline switch of receptor processing regulates commissural axon guidance in vertebrates. Genes Dev. 24, 396-410.

Nichols, E. L. and Smith, C. J. (2019). Pioneer axons employ Cajal's battering ram to enter the spinal cord. Nat. Commun. 10, 562. doi:10.1038/s41467-019-08421-9. 
Nicol, X., Hong, K. P. and Spitzer, N. C. (2011). Spatial and temporal second messenger codes for growth cone turning. Proc. Natl. Acad. Sci. U. S. A. 108, 13776-13781.

Pekarik, V., Bourikas, D., Miglino, N., Joset, P., Preiswerk, S. and Stoeckli, E. T. (2003). Screening for gene function in chicken embryo using RNAi and electroporation. Nat. Biotechnol. 21, 93-96.

Phan, K. D., Hazen, V. M., Frendo, M., Jia, Z. and Butler, S. J. (2010). The bone morphogenetic protein roof plate chemorepellent regulates the rate of commissural axonal growth. J. Neurosci. 30, 15430-15440.

Philipp, M., Niederkofler, V., Debrunner, M., Alther, T., Kunz, B. and Stoeckli, E. T. (2012). RabGDI controls axonal midline crossing by regulating Robo1 surface expression. Neural Dev. 7, 36.

Pignata, A., Ducuing, H., Boubakar, L., Gardette, T., Kindbeiter, K., Bozon, M., Tauszig-Delamasure, S., Falk, J., Thoumine, O. and Castellani, V. (2019). A Spatiotemporal Sequence of Sensitization to Slits and Semaphorins Orchestrates Commissural Axon Navigation. Cell Rep. 29, 347-362.e5.

Preitner, N., Quan, J., Li, X., Nielsen, F. C. and Flanagan, J. G. (2016). IMP2 axonal localization, RNA interactome, and function in the development of axon trajectories. Development. 143, 2753-2759.

Sanders, T. A., Llagostera, E. and Barna, M. (2013). Specialized filopodia direct longrange transport of $\mathrm{SHH}$ during vertebrate tissue patterning. Nature 497, 628-632.

Schindelin, J., Arganda-Carreras, I., Frise, E., Kaynig, V., Longair, M., Pietzsch, T., Preibisch, S., Rueden, C., Saalfeld, S., Schmid, B., et al. (2012). Fiji: An opensource platform for biological-image analysis. Nat. Methods 9, 676-682.

Squarzoni, P., Thion, M. S. and Garel, S. (2015). Neuronal and microglial regulators of cortical wiring: Usual and novel guideposts. Front. Neurosci. 9 :248. doi: 10.3389/fnins.2015.00248.

Stanganello, E. and Scholpp, S. (2016). Role of cytonemes in Wnt transport. J. Cell Sci. 129, 665-672.

Stoeckli, E. T. (2018). Understanding axon guidance: Are we nearly there yet? Development. 145, dev151415.

Vitriol, E. A. and Zheng, J. Q. (2012). Growth Cone Travel in Space and Time: The Cellular Ensemble of Cytoskeleton, Adhesion, and Membrane. Neuron 73, 10681081.

Wilson, N. H. and Stoeckli, E. T. (2011). Cell type specific, traceable gene silencing for functional gene analysis during vertebrate neural development. Nucleic Acids Res. 39, e133.

Wilson, N. H. and Stoeckli, E. T. (2012). In ovo electroporation of miRNA-based plasmids in the developing neural tube and assessment of phenotypes by Dil 
injection in open-book preparations. J. Vis. Exp.pii: 4384.

670

671

672

673

674

675

676

677

678

679

680

681

682

683

684

685

686
Wilson, N. H. and Stoeckli, E. T. (2013). Sonic Hedgehog regulates its own receptor on postcrossing commissural axons in a glypican1-dependent manner. Neuron 79, 478-491.

Yaginuma, H., Homma, S., Künzi, R. and Oppenheim, R. W. (1991). Pathfinding by growth cones of commissural interneurons in the chick embryo spinal cord: A light and electron microscopic study. J. Comp. Neurol. 304, 78-102.

Yoshioka, T. and Tanaka, O. (1989). Ultrastructural and cytochemical characterisation of the floor plate ependyma of the developing rat spinal cord. J. Anat. 165, 87-100.

Zisman, S., Marom, K., Avraham, O., Rinsky-Halivni, L., Gai, U., Kligun, G., Tzarfaty-Majar, V., Suzuki, T. and Klar, A. (2007). Proteolysis and membrane capture of F-spondin generates combinatorial guidance cues from a single molecule. J. Cell Biol. 178, 1237-1249.

Zou, Y. (2012). Does Planar Cell Polarity Signaling Steer Growth Cones? In Current Topics in Developmental Biology, pp. 141-160. 

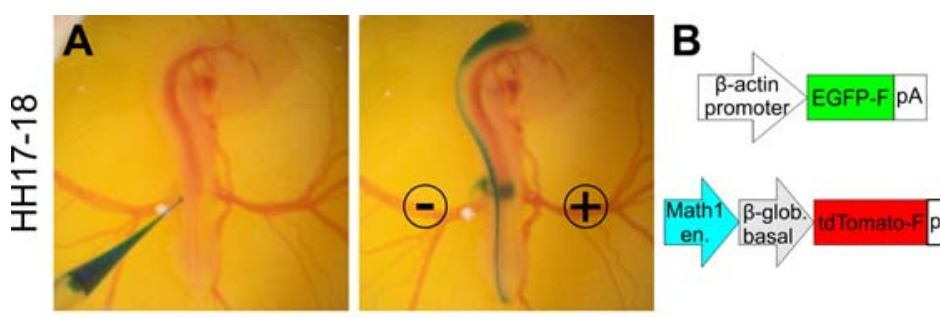

B-actin::EGFP-F Math1::tdTomato-F

Hoechst

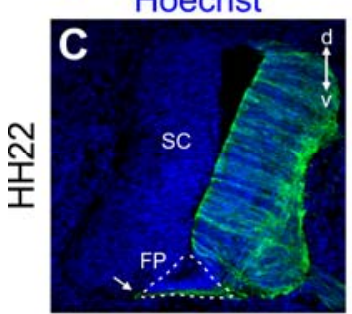

Hoechst
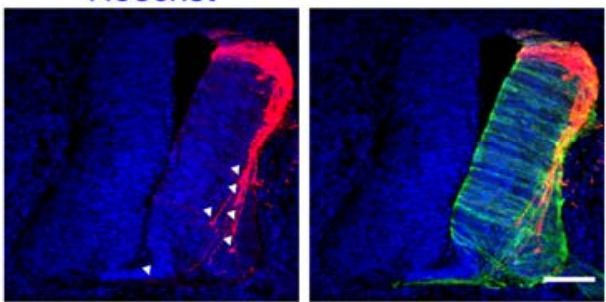

intact spinal cord

$\beta$-actin::EGFP-F

Brightfield Math1::tdTomato-F $\beta$-actin::EGFP-F
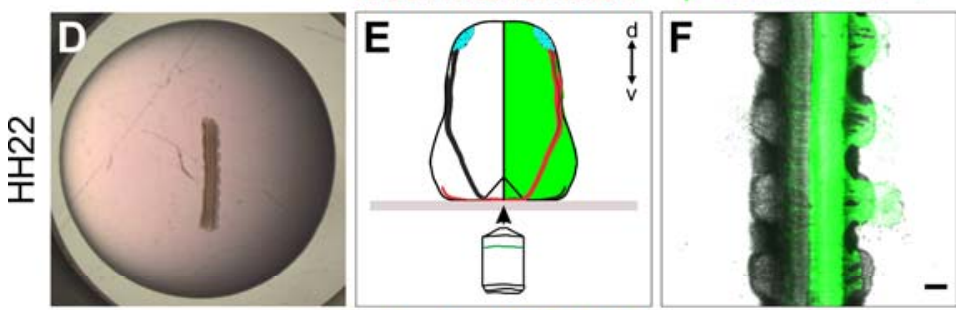

open-book

B-actin::EGFP-F

Brightfield

Math1::tdTomato-F $\beta$-actin::EGFP-F
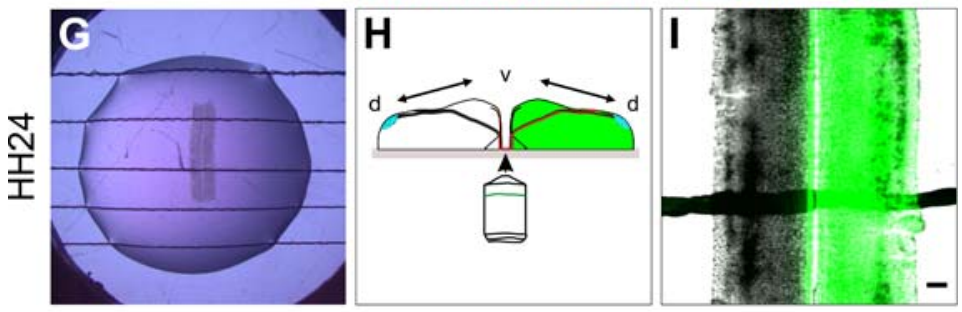

Figure1, Dumoulin et al.

Fig. 1. Labeling strategy for dl1 interneurons and spinal cord culture systems. (AC) In ovo injection and electroporation of a plasmid mix to specifically label dl1 interneurons. (A) The plasmid mix was injected into the central canal of the spinal cord of $\mathrm{HH} 17-18$ chicken embryo in ovo, followed by unilateral electroporation. (B) Plasmid 692 constructs injected to target all cells ( $\beta$-actin::EGFP-F) and dl1 interneurons (Math1::tdTomato-F). en., enhancer; $\beta$-glob., $\beta$-globin. (C) Immunostaining of a

694 transverse cryosection of a $\mathrm{HH} 22$ spinal cord taken from an embryo sacrificed one day 695 after electroporation with the plasmids indicated in (B). At this stage, most dl1 growth cones were approaching the FP area, but none of them had crossed it yet (white 
697 arrowheads). However, a substantial number of Math1-negative, but EGFP-F-

698 expressing commissural axons of more ventral populations had already crossed the FP

699 at HH22 (arrow). (D-F) Intact spinal cord culture. (D) Intact spinal cords of embryos

700 injected and electroporated one day earlier were embedded with the ventral side down

701 in a drop of low-melting agarose. (E) The ventral spinal cord area was imaged with an

702 inverted spinning disk microscope. The green-colored hemisphere represents the

703 electroporated side of the spinal cord. (F) Low magnification overview of a spinal cord

704 visualized with this set-up with cells expressing EGFP-F under the $\beta$-actin promoter on

705 one side merged with the brightfield image. (G-I) Culture of an open-book preparation of

706 a spinal cord. (G) Intact open-book preparations of HH24 spinal cords dissected from

707 embryos injected and electroporated about one and a half day earlier were embedded

708 with the apical side down in an agarose drop with strings to hold it in place. $(H)$ The

709 midline area was visualized with the same inverted spinning disk microscope as above.

710 The green-colored hemisphere represents the electroporated side of the spinal cord. (I)

711 Low magnification overview of a spinal cord visualized with this set-up with cells

712 expressing EGFP-F under the $\beta$-actin promoter on one side merged with the brightfield

713 image. SC, spinal cord; d, dorsal; v, ventral. Scale bars: $100 \mu \mathrm{m}$. 


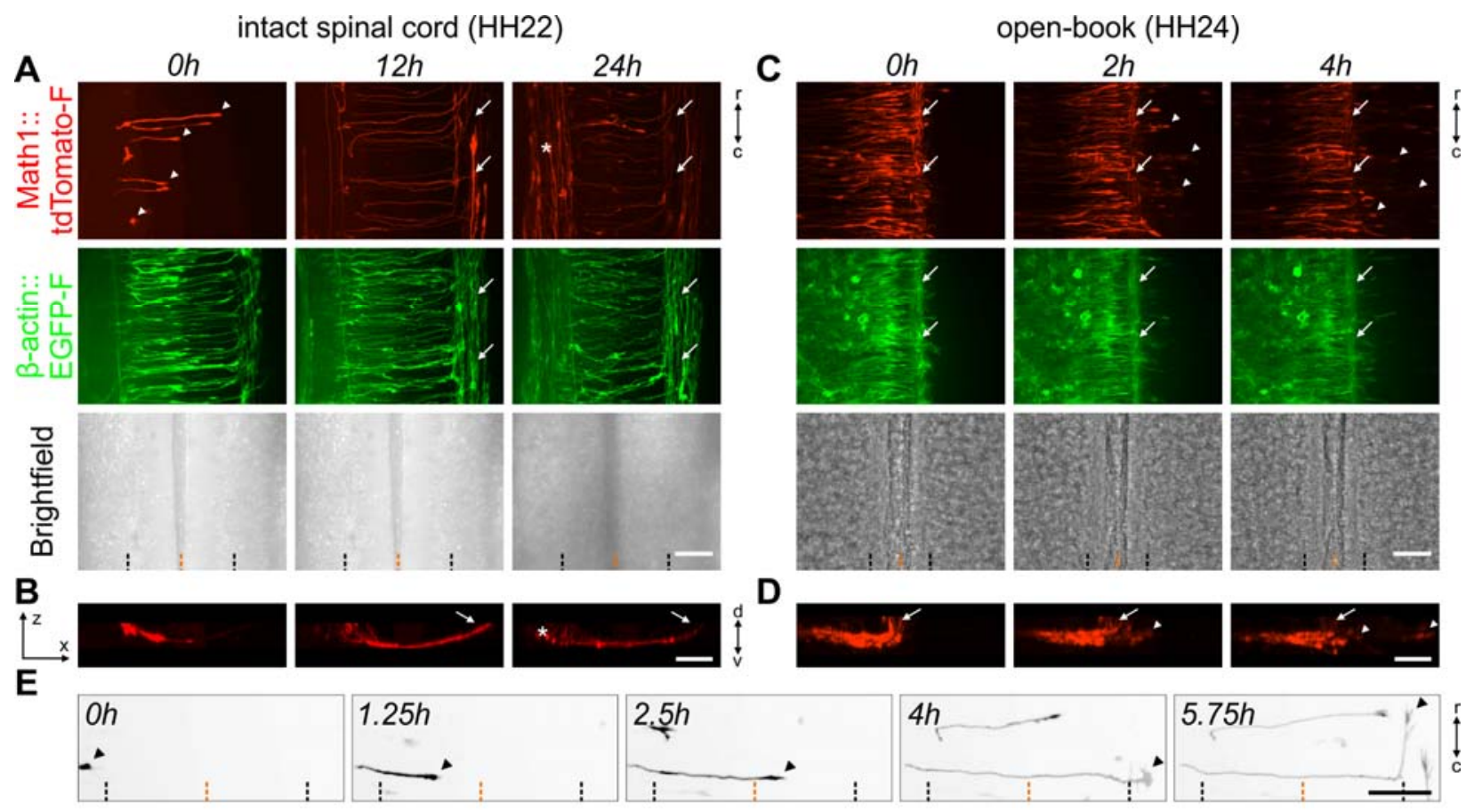

715

716

Figure 2, Dumoulin et al. Fig. 2. Live imaging of cultured intact spinal cords allowed for the visualization of dl1 axons during floor-plate crossing and navigation into the longitudinal axis. (A) 24-h time-lapse recording showed that Math1-positive dl1 commissural axons could cross the FP (white arrowheads), turn anteriorly, as expected, and form the contralateral ventral funiculus (white arrows) in cultured intact $\mathrm{HH} 22$ spinal cords. The asterisk indicates a population of Math1-positive ipsilateral axons. (B) Transversal view of a region of interest from the time-lapse recording shown in $(A)$, highlighting the trajectory of dl1 axons and the formation of the commissure. The white arrow indicates the position of the contralateral ventral funiculus. The white asterisk labels ipsilateral axons. (C) 4-h time-lapse recording showing Math1-positive dl1 commissural axons in a cultured open-book preparation of a HH24 spinal cord. Note that within less than $2 \mathrm{~h}$ in culture the majority of dl1 commissural axons were overshooting the contralateral FP boundary and growing straight into the contralateral side after having crossed the FP (white arrowheads). White arrows indicate the contralateral ventral funiculus. (D) Transversal view of a region of interest from the time-lapse sequence shown in (C) highlighting the aberrant trajectory of dl1 commissural axons (white arrowheads) growing straight past the contralateral ventral funiculus (white arrow). (E) Single dl1 
733 growth cones (black arrowheads) could be tracked crossing the FP, exiting it and 734 turning rostrally. Math1-positive axons are now shown in black. Black and orange 735 dashed lines indicate FP boundaries and the midline, respectively. $d$, dorsal; v, ventral; $736 r$, rostral; c, caudal. Scale bars: $50 \mu \mathrm{m}$.

737 

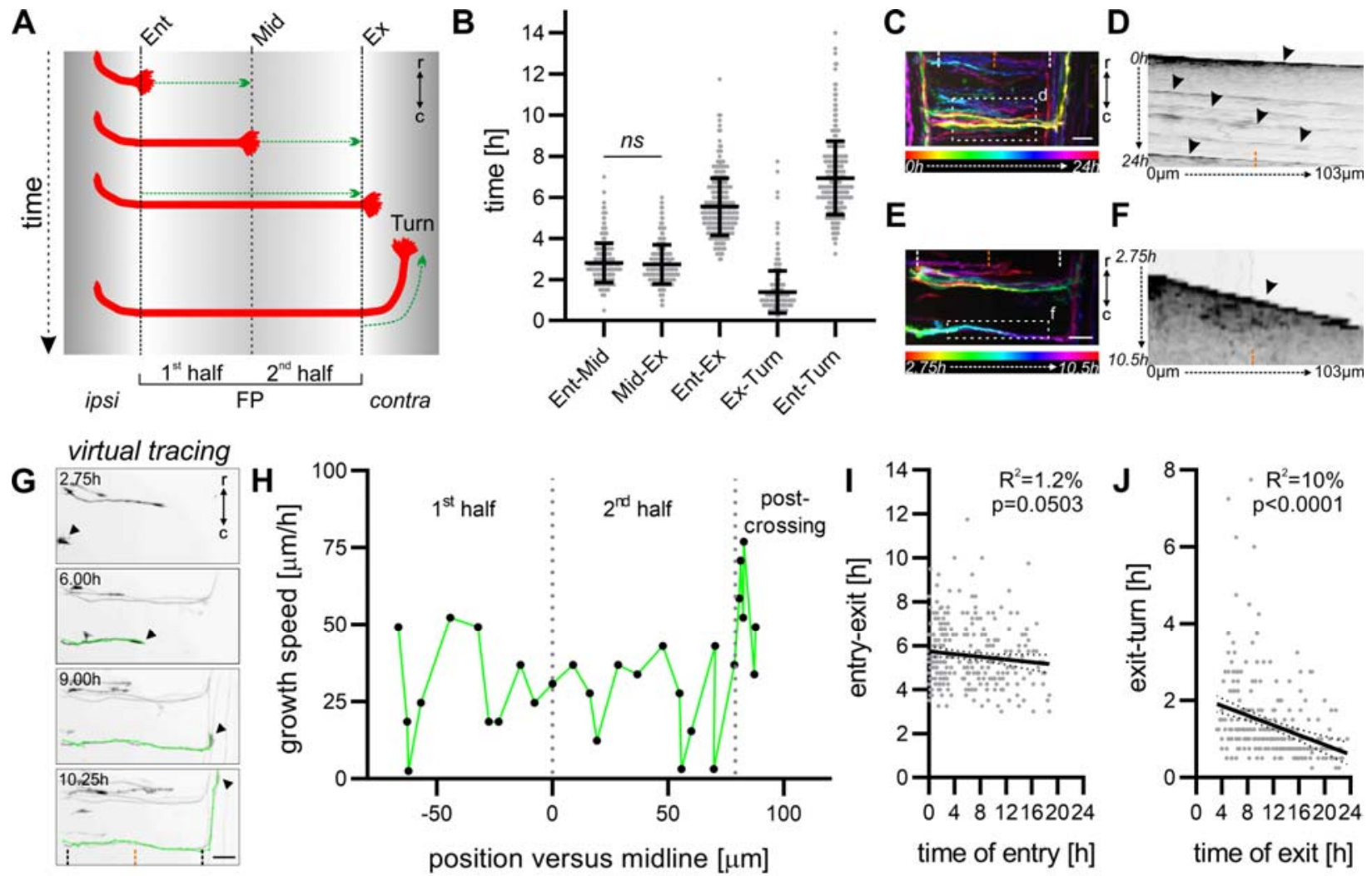

Figure 3, Dumoulin et al.

741

742

743

744

745

746

747

748

749

750

751

752

753

754

Fig. 3. Characterization of the timing of midline crossing by dl1 commissural axons. (A) Schematic depicting how the timing of midline crossing for individual dl1 axons was measured for each segment of interest. (B) Graph showing the average time a growth cone spent in each segment shown in (A) $\left(N_{\text {embryos }}=7 ; n_{\text {axons }}=298\right)$. There was no significant difference in the time taken by dl1 axons to cross the first versus the second half of the FP ( $\mathrm{p} \geq 0.05$, paired two-tailed Wilcoxon test). (C) Temporal-color code projection of a 24-h time-lapse recording. (D) Kymograph of the 24-h time-lapse recording in the region of interest within the FP shown in (C). Several axons crossing the FP at different times can be visualized (black arrowheads). (E) Temporal-color code projection of 7.75-h time-lapse recording segment. (F) Kymograph of the 7.75-h timelapse recording segment in the region of interest within the FP shown in (E). Within this time segment only one axon crossed the FP and could be visualized in the kymograph (black arrowhead). White and orange dashed lines represent the FP boundaries and the midline, respectively. (G) A virtual tracing tool (shown in green) was used to extract the 
755 velocity of the growth cones (black arrowheads) at each time point during midline 756 crossing at the single axon level. The same axon is shown in (E) and (G). Black dashed 757 lines and the orange dashed lines represent the FP boundaries and midline, 758 respectively. $(H)$ The growth speed of the axon shown in $(G)$ could be extracted and 759 plotted against the position of the growth cone in the FP. Dotted grey lines represent the 760 time at which the axon crossed the midline or exited the FP. $(I, J)$ The time of crossing 761 the FP (entry-exit) or of turning (exit-turn) for each axons measured in (B) was plotted 762 against the time of FP entry and exit of the growth cone, respectively. (I) The time axons 763 took to cross the FP appeared to decrease over time although this was not significant. 764 (J) The time axons took to turn after exiting the FP decreased significantly over time. 765 ipsi, ipsilateral; contra, contralateral; Ent, entry; Mid, midline; Ex, exit; r, rostral; c, 766 caudal. Scale bars: $25 \mu \mathrm{m}$. 

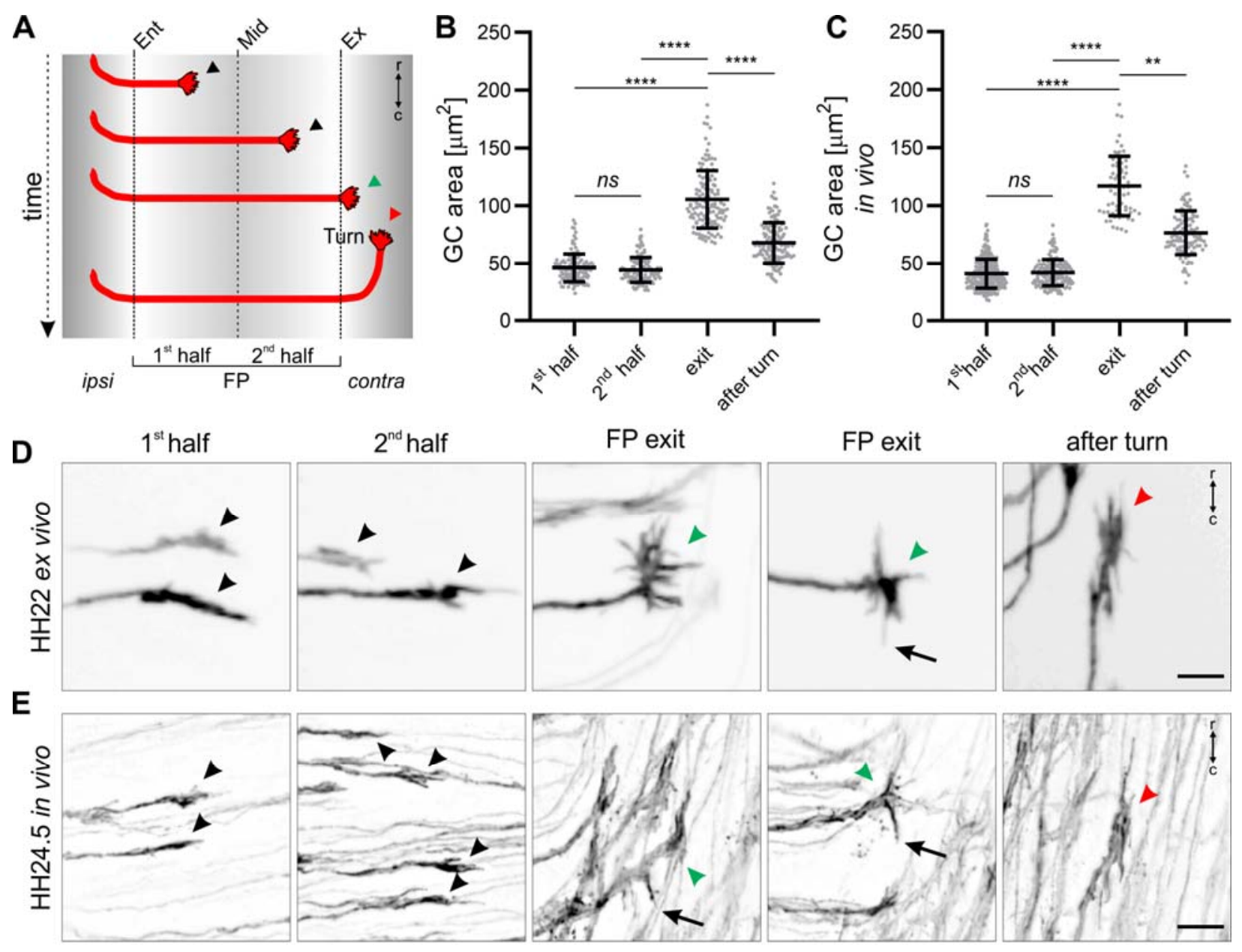

Fig. 4. Live imaging of intact spinal cords revealed dl1 growth cone morphologies at chosen time points. (A) Schematic depicting where the growth cone area of individual dl1 axons was measured for (B) and (C). (B) Average growth cone areas were measured from 24-h time-lapse recordings of dl1 axons crossing the midline

773 ( $\left.\mathrm{N}_{\text {embryos }}=7 ; \mathrm{n}_{\text {growth cones }}=127\right)$. No significant difference in the area of growth cones was 774 found between the first and second half of the FP. However, growth cones were 775 significantly larger at the exit site but then again reduced in size after having turned 776 rostrally (paired Friedman test with Dunn's multiple-comparisons test). (C) Average 777 growth cone areas were measured in vivo from fixed HH23-25 spinal cords ( $\mathrm{N}_{\text {embryos }}=8$;

$778 \mathrm{n}_{\text {growth cones }}=285\left(1^{\text {st }}\right.$ half), 153 ( $2^{\text {nd }}$ half), 68 (exit) and 102 (after turn). The relationship 779 between the average growth cone area and the position in the FP corroborated results 780 using the ex vivo culture system shown in (B) (unpaired Kruskal-Wallis test with Dunn's 
781 multiple-comparisons test). (D,E) Examples corroborating the similarities in growth cone 782 morphology ex vivo and in vivo in the FP (black arrowheads), at the exit site (green 783 arrowheads) and after rostral turn (red arrowheads). At the exit site, growth cones were 784 spiky with always some filopodia pointing caudally just before rostral turn, a feature that 785 was also observed in vivo (black arrows). ipsi, ipsilateral; contra, contralateral; r, rostral;

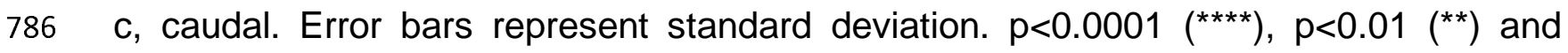
$787 \mathrm{p} \geq 0.05$ (ns) for all tests. Scale bars: $10 \mu \mathrm{m}$. 

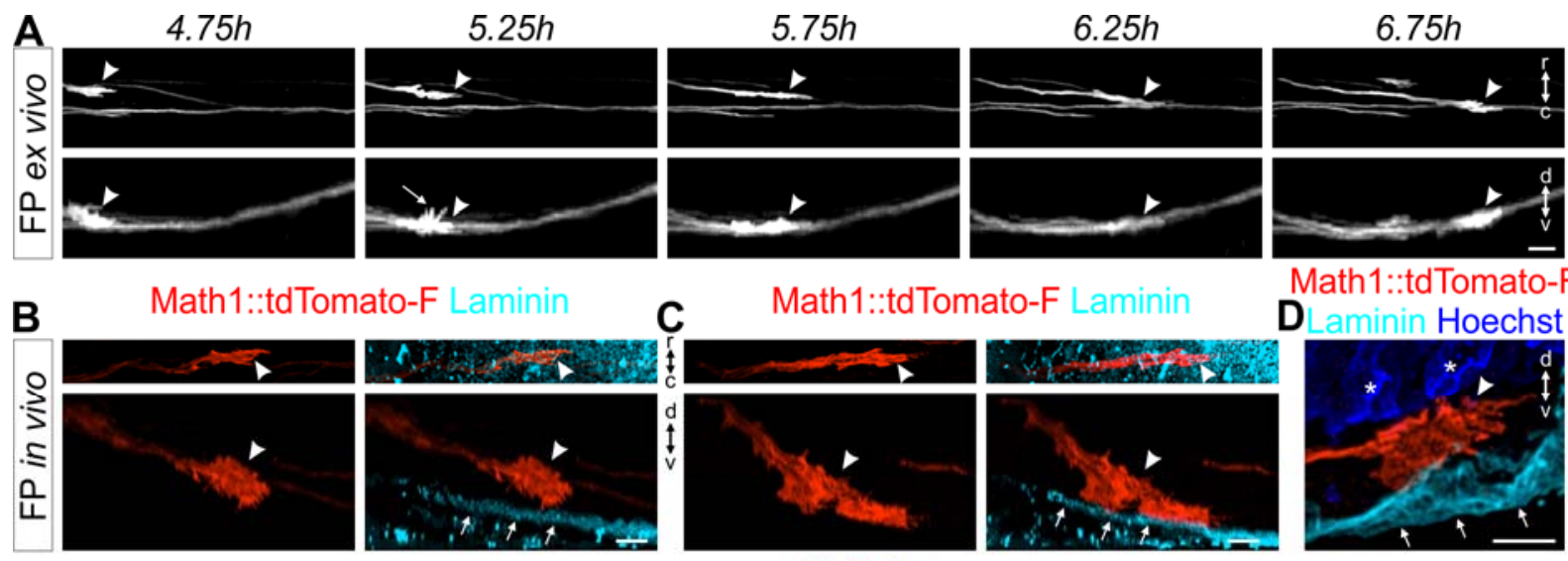

Math1::tdTomato-F

DLaminin Hoechst
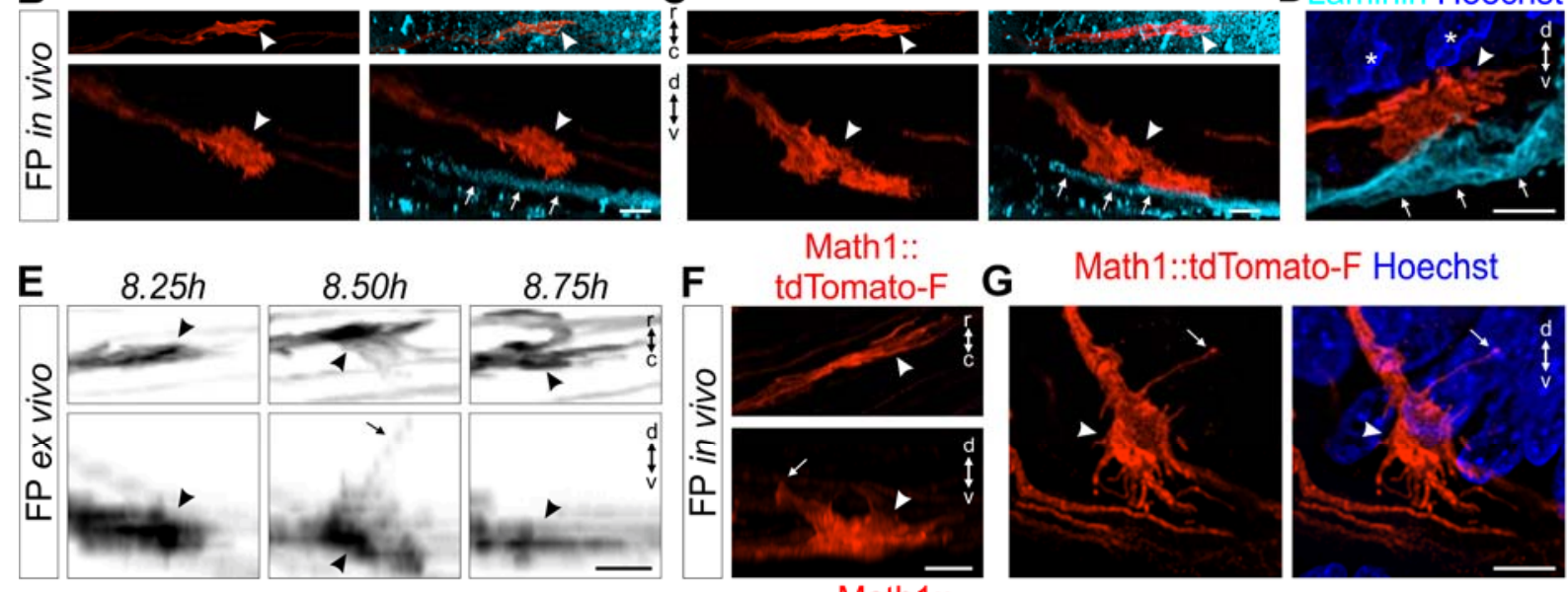

Math1::tdTomato-F Hoechst
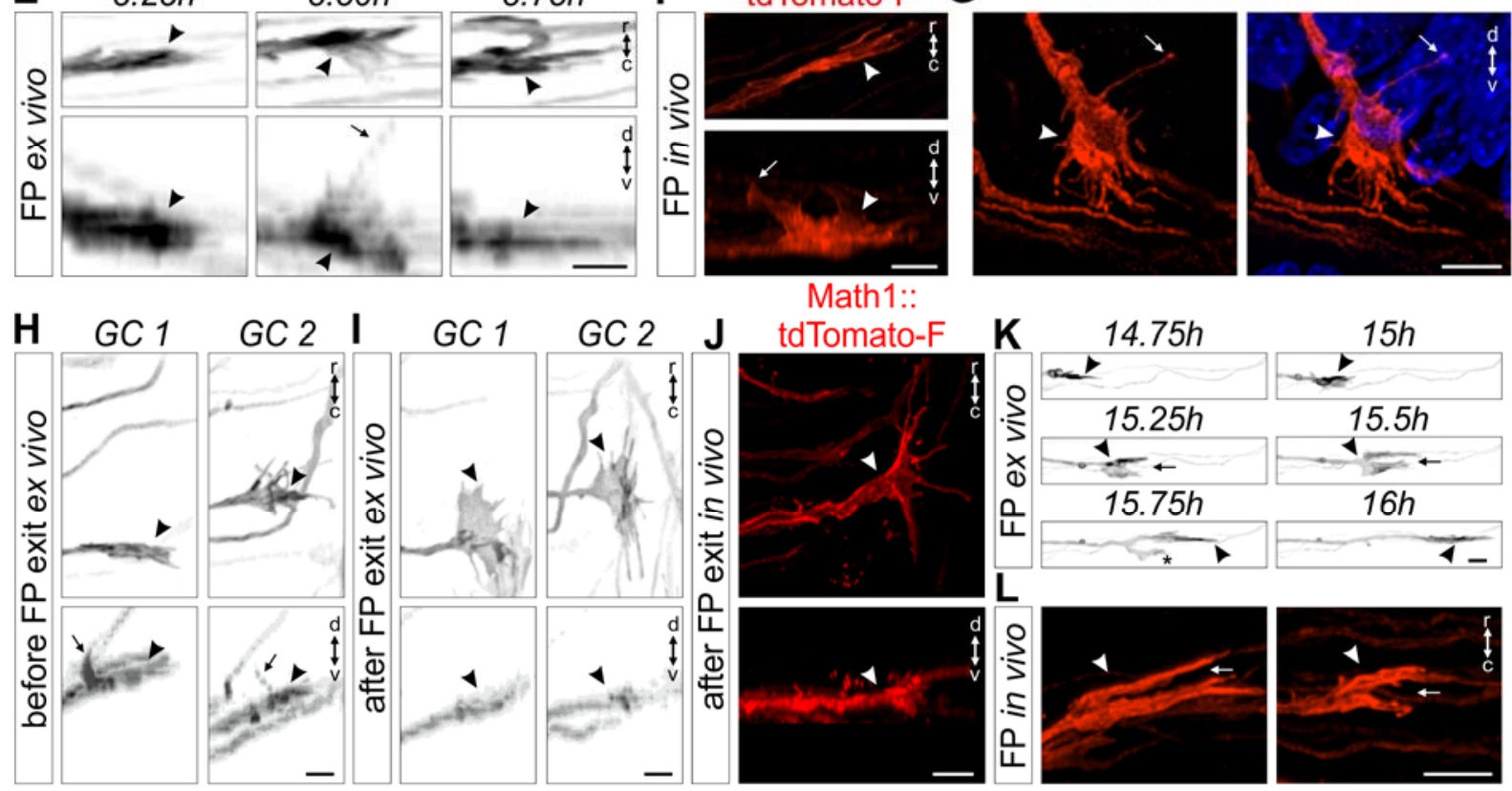

Figure 5, Dumoulin et al.

Fig. 5. High magnification live imaging of dl1 commissural axons unraveled their orientation and activities at choice-points. (A) A Math1::tdTomato-F-positive growth cone (white arrowheads) crossing the FP shown in the rostro-caudal and dorso-ventral axis. White arrow shows the dorso-ventral orientation of the growth cone with some filopodia extended towards the apical FP. (B,C) Whole-mount immunostaining of Math1::tdTomato-F-positive dl1 growth cones in the FP at HH24.5 showed their dorsoventral orientation in vivo (white arrowheads). The basal lamina was stained for laminin

797 (white arrows). (D) A Math1::tdTomato-F-positive dl1 growth cone in the FP (white 798 arrowhead) at $\mathrm{HH} 22$ showed its dorso-ventral orientation in vivo (white arrowheads). 
799 The basal lamina was stained for laminin (white arrows) and nuclei with Hoechst. White 800 asterisk show nuclei from the FP cells. (E) Three consecutive snapshots from a time801 lapse sequence showing the dorso-ventral activity of a Math1::tdTomato-F-positive 802 growth cone crossing the FP (black arrowheads) with a long protrusion growing toward 803 the FP soma level (black arrow). (F,G) A long protrusion growing towards the apical FP 804 cell soma area (white arrows) could be also observed in dl1 growth cones crossing the 805 FP in vivo (white arrowheads) after whole-mount staining of a HH24 spinal cord (F) or 806 immunostaining on a HH22 spinal cord transverse section $(\mathrm{G})$. $(\mathrm{H})$ Example of two 807 growth cones extracted from a time-lapse recording (black arrowheads) showing dorsal 808 activity with a long protrusion (black arrows) growing towards the FP soma area just 809 before exiting the FP. (I) The same growth cones shown in $(\mathrm{H})$ after exiting the FP 810 underwent a $90^{\circ}$ change in their orientation. They now were thin in the dorso-ventral 811 axis and enlarged in the rostro-caudal axis (black arrowheads). (J) HH24.5 whole-mount 812 immunostaining of Math1::tdTomato-F-positive dl1 growth cone at the FP exit site 813 showed that the orientation of post-crossing growth cones in vivo were the same as 814 observed by live imaging (white arrowheads). (K) Example of a Math1::tdTomato-F815 positive dl1 growth cone (black arrowhead) transiently splitting (black arrow) while 816 crossing the FP. One branch always retracted (black asterisk) while the other one 817 continued to grow straight to the contralateral side. (L) Split growth cones (white arrow) 818 of Math1::tdTomato-F-positive dl1 axons (white arrowheads) could be observed in vivo 819 after whole-mount staining of HH24.5 spinal cords. GC, growth cone; r, rostral; c, 820 caudal; d, dorsal; v, ventral. Scale bars: $10 \mu \mathrm{m}(\mathrm{A})$ and $5 \mu \mathrm{m}(\mathrm{B}-\mathrm{L})$. 


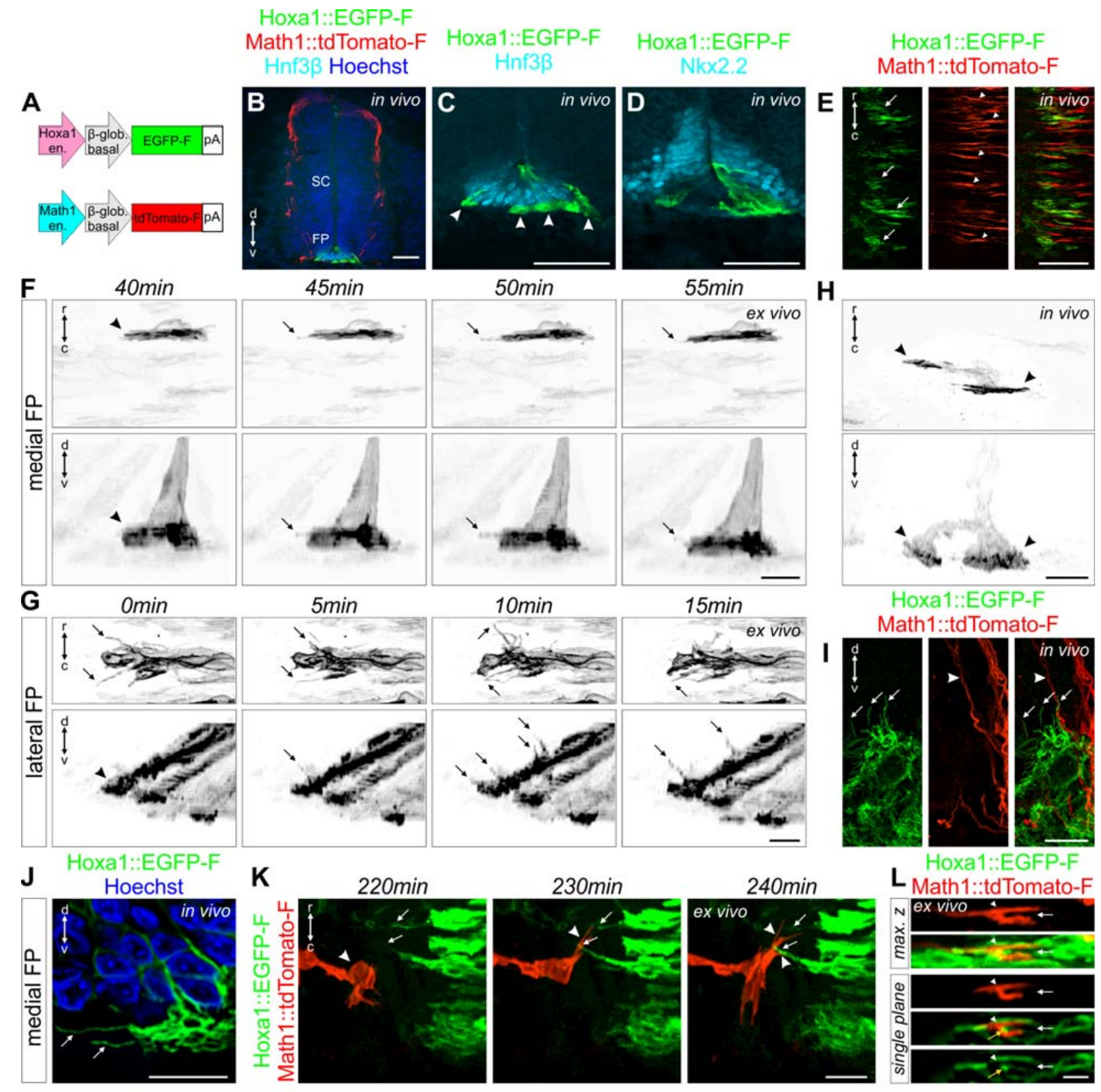

Figure 6, Dumoulin et al.

824 Fig. 6. Live imaging of floor-plate cells during midline crossing shed light on their

825 orientation and dynamics. (A) Schematic depicting plasmids that were electroporated

826 at $\mathrm{HH} 17-18$ to visualize FP cells (Hoxa1 plasmid) together with dl1 axons (Math1

827 plasmid). (B) Immunostaining of $\mathrm{HH} 22$ spinal cord cryosections revealed the restricted

828 expression of Hoxa1::EGFP-F in FP cells co-stained for Hnf3 $\beta$. (C) Higher magnification

829 of the section shown in (B) clearly showing Hnf3ß-positive FP cells expressing EGFP-F.

830 Bulky FP basal feet could be observed in the commissure (white arrowheads). (D) 
831 Immunostaining of $\mathrm{HH} 22$ spinal cord cryosections showing that EGFP-F expression

832 driven by Hoxa1 enhancer was mostly absent in Nkx2.2-positive cells flanking the FP.

833 (E) Whole-mount immunostaining of Math1::tdTomato-F-positive dl1 axons and 834 Hoxa1::EGFP-F-positive FP cells revealed and alignment between dl1 growth cones 835 (white arrowheads) and basal feet of FP cells (white arrows) in the commissure. (F) 836 Example of a time-lapse recording of a single medial Hoxa1::EGFP-F-positive FP cell to 837 reveal the geometry of its basal foot that was thin along the rostro-caudal axis but 838 enlarged in the dorso-ventral axis (black arrowheads). The basal foot was highly 839 dynamic with protrusions sprouting out in the directions of axonal growth in the 840 commissure (black arrows). (G) Example of a time-lapse recording of lateral 841 Hoxa1::EGFP-F-positive FP cells showing a very high activity of their basal feet with 842 highly dynamic protrusions growing towards the arriving pre-crossing axons (black 843 arrows). (H) Whole-mount immunostaining of a single medial Hoxa1::EGFP-F-positive 844 FP cell in vivo at HH24.5 showing similar shape (black arrowheads) as observed by live 845 imaging. Note that this FP cells contained two feet (black arrowheads). (I) 846 Immunostaining of a HH22 spinal cord cryosection revealed that lateral Hoxa1::EGFP-

847 F-positive FP cells also formed protrusions growing towards the pre-crossing axons in 848 vivo (white arrows) where Math1::tdTomato-F-positive axons enter the FP (white 849 arrowheads). (J) Similar protrusions were observed in HH22 medial FP basal feet in the 850 commissure in vivo using immunostaining of cryosections (white arrows). The picture 851 shows a single plane extracted from a Z-stack. (K) Snapshots extracted from a time852 lapse sequence of a Math1::tdTomato-F-positive dl1 growth cone interacting (white 853 arrowheads) with protrusions from Hoxa1::EGFP-F-positive FP basal feet (white arrows) 854 before entering the FP. (L) Single snapshot from a time-lapse recording sequence 855 showing maximum $Z$ projection and single plane pictures of a transiently splitting (white 856 arrow) Math1::tdTomato-F-positive dl1 growth cone (white arrowhead) in the FP with 857 basal feet structures in between the two split branches (yellow arrowhead). SC, spinal 858 cord; r, rostral; c, caudal; d, dorsal; v, ventral. Scale bars: $50 \mu m(A), 25 \mu m(B)$ and 10 $859 \mu \mathrm{m}(\mathrm{C}-\mathrm{L})$. 


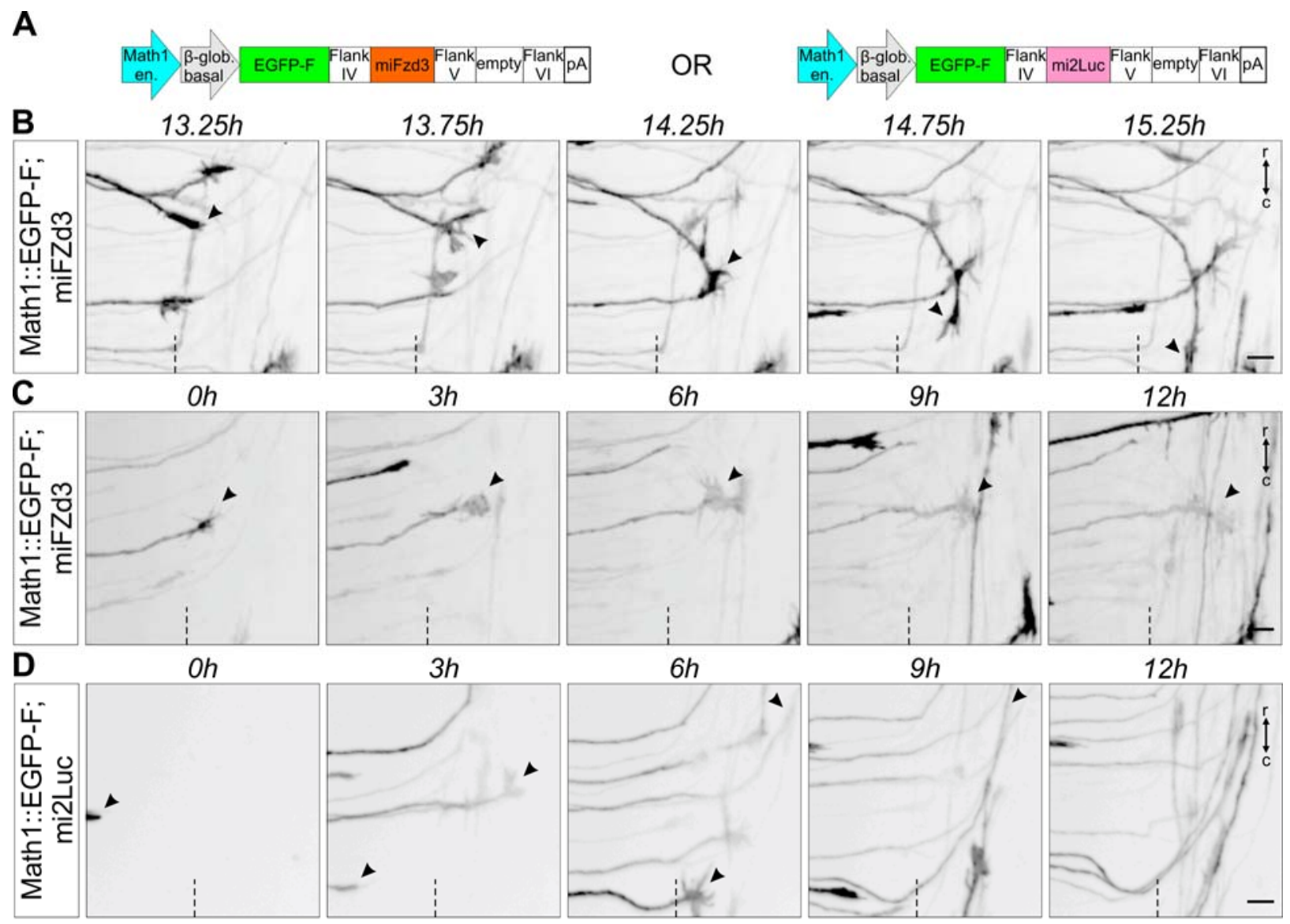

Fig. 7. Live imaging after dl1 neuron-specific knockdown can be used to visualize mutant axons in intact spinal cord preparations. (A) Schematics depicting the plasmid constructs used to knockdown Fzd3 in dl1 neurons. A plasmid expressing a microRNA against Luciferase (mi2Luc) was used as a control. (B) Time-lapse sequence showing a dl1 commissural axon turning caudally instead of rostrally at the FP contralateral border after silencing Fzd3 (black arrowheads). (C) Time-lapse sequence showing a dl1 commissural axon stalling at the contralateral FP border after silencing 870 Fzd3 (black arrowheads). The growth cone kept remodeling but was not able to turn in either direction. (D) Time-lapse sequence showing dl1 axons expressing a microRNA

872 against luciferase. These axons were not impacted and after exiting the FP they all 873 turned rostrally (black arrowheads). Dashed black line represents the FP exit site. $r$, rostral; c, caudal. Scale bars: $10 \mu \mathrm{m}$. 


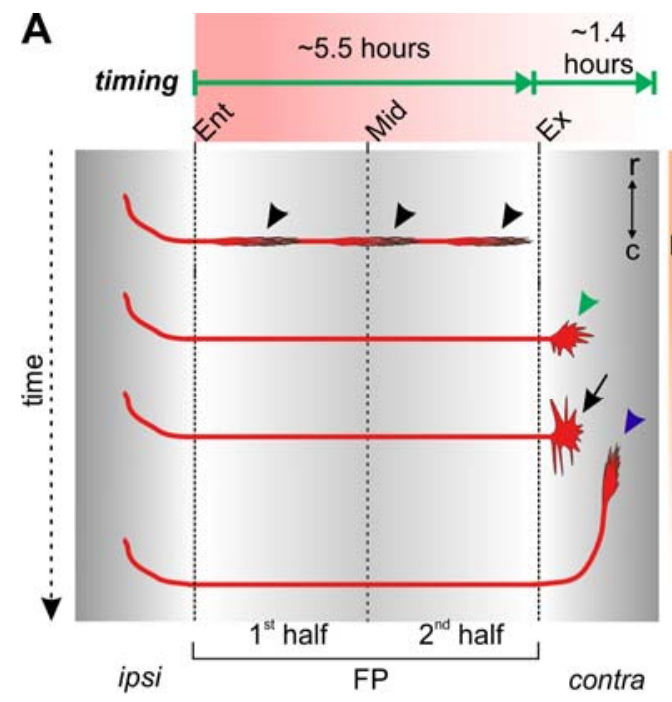

877

878

879

880

881

882

883

884

885

886

887

888

889

890

891

892

893

894

895

896

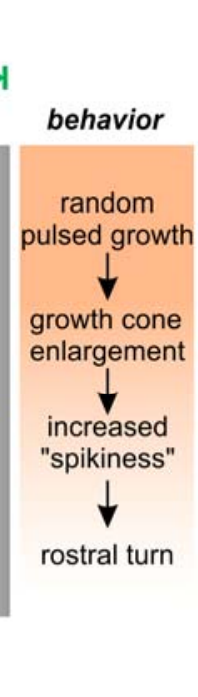

D FP exit

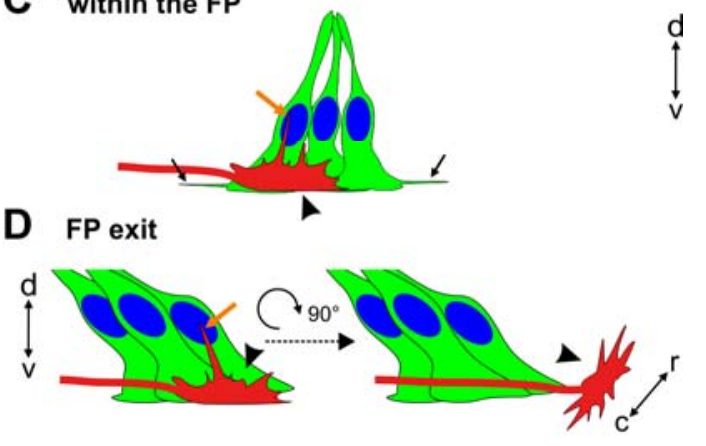

Figure 8, Dumoulin et al.

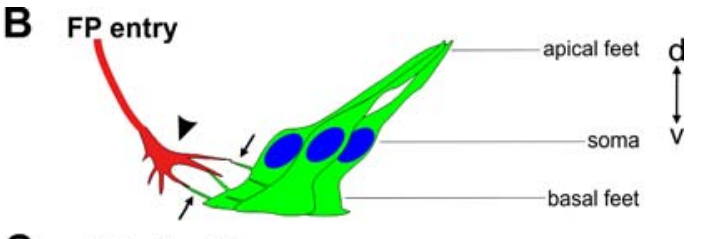

C within the FP

Fig. 8. Cartoon depicting the midline crossing characteristics of dl1 axons based on data extracted from our ex vivo culture system. (A) On average, it took dl1 axons 5.6 hours to cross the midline. Growth cones showed a random pulsed growth and had a thin shape in the growth direction (black arrowheads). At the FP exit site, dl1 growth cones were first enlarged (green arrowhead), then extended filopodia along the longitudinal axis (black arrow) right before turning rostrally (blue arrowhead). After arriving at the exit site of the FP, it took dl1 axons on average about 1.4 hours to turn rostrally. In fact, the first exiting dl1 axons took longer to turn rostrally than the late exiting ones. (B-D) Live imaging of intact spinal cords ex ovo using a high magnification objective shed light on dl1 growth cone orientation, FP morphology and dynamics during midline crossing. (B) While dl1 growth cones (black arrowhead) approached the FP, basal feet of lateral FP cells sent protrusions towards them and eventually interacted with them (black arrows). (C) When dl1 growth cones crossed the FP (black arrowhead), their dorso-ventral orientation aligned perfectly with the orientation of basal feet of medial FP cells. While basal feet of FP cells sent protrusions in axonal growth direction (black arrows), dl1 growth cones sent long filopodia in direction of the apical FP, towards the FP cell soma (orange arrow). (D) Just before exiting the FP, dl1 growth cones showed dorso-ventral activity with a long protrusion growing towards the FP soma (orange arrow) area followed by a $90^{\circ}$ change in their orientation to become 
bioRxiv preprint doi: https://doi.org/10.1101/2020.07.20.211995; this version posted July 20, 2020. The copyright holder for this preprint (which

was not certified by peer review) is the author/funder, who has granted bioRxiv a license to display the preprint in perpetuity. It is made available under aCC-BY-NC-ND 4.0 International license.

897 flattened in the dorso-ventral axis and enlarged in the longitudinal axis (black 898 arrowhead). Ent, entry; Mid, midline; Ex, exit; r, rostral; c, caudal; ipsi, ipsilateral; contra, 899 contralateral.

900 
Movie 1. 24-hour time-lapse recording of the ex vivo cultured intact spinal cord

903

904

905

906

907

908

909

910

911

912

913

914

915

916

917

918

919

920

921

922

923

924

925

926

927 shown in Fig. 2A. Math1::tdTomato-F-positive dl1 axons (red), $\beta$-actin::EGFP-Fpositive axons and cells (green) and brightfield maximum projections are shown in parallel. One z-stack was taken every 15 minutes for each channel. White and orange dashed lines indicate FP boundaries and the midline, respectively. Rostral is up.

Movie 2. 8-hour segment of a time-lapse recording of an intact spinal cord cultured ex vivo. Math1::tdTomato-F-positive dl1 axons are shown in black. Maximum projections of z-stacks taken every 15 minutes are represented as well as 3D rotations at different time points (at FP entry, during FP crossing, at FP exit and after rostral turn). Black and orange dashed lines indicate FP boundaries and midline, respectively. Rostral is up.

Movie 3. 4-hour time-lapse recording of the ex vivo cultured open-book preparation of the spinal cord shown in Fig. 2C,D. Math1::tdTomato-F-positive dl1 axons (red), $\beta$-actin::EGFP-F-positive axons and cells (green) and brightfield maximum projections are shown in parallel. One z-stack was taken every 15 minutes for each channel. White and orange dashed lines indicate FP boundaries and the midline, respectively. Rostral is up.

Movie 4. 24-hour time-lapse recording of an ex vivo cultured intact spinal cord used for kymographic analysis in Fig. 3D. Math1::tdTomato-F-positive dl1 axons are shown in black. Maximum projections of z-stacks taken every 15 minutes are represented. Black and orange dashed lines indicate FP boundaries and midline, respectively. Rostral is up. 
Movie 5. 7.75-h time-lapse recording of the segment shown in Fig. 3G. This movie shows the virtual tracing of the growth cone (black arrowhead) at each time point revealing the instantaneous growth speed. Math1::tdTomato-F-positive dl1 axons are shown in black. Maximum projections of z-stacks taken every 15 minutes are represented. Black and orange dashed lines indicate FP boundaries and midline, respectively. Rostral is up.

Movie 6. Example of growth cone enlargement at the floor-plate exit site. Growth cone of a Math1::tdTomato-F-positive dl1 commissural axon (black arrowheads) exiting the FP (black dashed line) and becoming spiky with many filopodia before turning rostrally (black arrow). Rostral is up.

Movie 7. Example of growth cone enlargement at the floor-plate exit site. Two growth cones of Math1::tdTomato-F-positive dl1 commissural axons (black arrowheads) exiting the FP (black dashed line) and becoming spiky with filopodia growing in rostral and caudal direction before turning rostrally (black arrows). Rostral is up.

Movie 8. Example of growth cone enlargement and transient splitting at the floorplate exit site. Growth cone of a Math1::tdTomato-F-positive dl1 commissural axon (black arrowheads) exiting the FP (black dashed line), becoming spiky with a lot of filopodia and transiently splitting before turning rostrally (black arrow). Rostral is up.

\section{Movie 9. 24-hour time-lapse recording with high magnification objective of} cultured intact spinal cord. Math1::tdTomato-F-positive dl1 axons are shown in black. Maximum projections of z-stacks taken every 15 minutes are represented. Dashed lines represent FP boundaries. Rostral is up. 
Movie 10. Time-lapse recording within the floor plate uncovered dl1 growth cones orientation. Math1::tdTomato-F-positive dl1 growth cone (white arrowhead) crossing the FP showed a dorso-ventral orientation with filopodia activities in the dorso-ventral axis (white arrows). Maximum projections of z-stacks (rostro-caudal and dorso-ventral axis) taken every 15 minutes are represented as well as a 3D rotation. $r$, rostral; c, caudal; d, dorsal; v, ventral.

Movie 11. dl1 growth cones showed the same dorso-ventral orientation in the floor plate in vivo (whole-mount staining of HH24.5 intact spinal cord). 3D representation of two Math1::tdTomato-F-positive dl1 growth cones (white arrowheads) showing little extension in the rostro-caudal axis but enlarged size in the dorso-ventral axis. The basal lamina was stained for laminin (shown in cyan, white arrows). r, rostral; c, caudal; d, dorsal; v, ventral.

Movie 12. Early crossing dl1 growth cones showed the same dorso-ventral orientation in the floor plate in vivo as observed ex vivo (immunostaining of HH22 spinal cord cross-sections). 3D representation of a Math1::tdTomato-F-positive dl1 growth cones (white arrowheads) showing little extension in the rostro-caudal axis but enlarged size in the dorso-ventral axis. The basal lamina was stained for laminin (shown in cyan) and nuclei counter-stained with Hoechst (shown in blue). r, rostral; c, caudal; d, dorsal; v, ventral.

Movie 13. 14-hour time-lapse recording of dl1 axons crossing the floor-plate. Rostro-caudal, dorso-ventral and 3D representations of Math1::tdTomato-F-positive dl1 axons crossing the FP. dl1 growth cones transiently split in the FP (black asterisks) and showed filopodial extensions towards the FP cell soma area (black arrowheads). $r$, rostral; c, caudal; d, dorsal; v, ventral. 
Movie 14. Detailed analysis of dl1 growth cone shape at the floor-plate exit site in real time. Sequence from a time-lapse recording showing two Math1::tdTomato-Fpositive dl1 commissural growth cones exiting the FP (black arrowheads). Both sent a protrusion towards the FP cell soma just before exiting (black arrows) and changed their orientation upon exiting the FP by $90^{\circ}$ to adopt an enlarged size in the longitudinal axis. Dashed line represents the FP exit. r, rostral; c, caudal; d, dorsal; v, ventral.

Movie 15. dl1 growth cone at the floor-plate exit site of a HH24.5 spinal cord in vivo. Example of a Math1::tdTomato-F-positive growth cone (white arrowhead) with little extension along the dorso-ventral axis but enlarged in the rostro-caudal axis. $r$, rostral; c, caudal; d, dorsal; v, ventral.

Movie 16. dl1 growth cone transiently split in the floor plate. Sequence from a timelapse recording showing a Math1::tdTomato-F-positive dl1 commissural growth cone crossing the FP (black arrowhead) and transiently splitting (black arrowhead). Note that one branch retracted (black asterisk) while the other continued to grow straight towards the contralateral side (black arrowhead). Rostral is up.

\section{Movie 17. Time-lapse recording sequence of a Hoxa1::EGFP-F-positive medial} floor plate cell. Live imaging of a medial FP cell showed that it had a thin basal foot in the rostro-caudal axis. In the dorso-ventral axis to foot was large. The FP cell extended highly dynamic protrusions from its foot in both directions of axonal growth (black arrows). r, rostral; c, caudal; d, dorsal; v, ventral.

\section{Movie 18. Time-lapse recording sequence of Hoxa1::EGFP-F-positive lateral floor-} plate cells. Live imaging of lateral FP cells revealed a very high activity of their basal feet with highly dynamic protrusions sprouting out in the direction of the arriving precrossing axons (black arrows). r, rostral; c, caudal; d, dorsal; v, ventral. 

in green) with two thin basal feet in the rostro-caudal axis that were enlarged in the dorso-ventral axis at the level of the commissure (white arrows). The orientation of basal feet was aligned to a Math1::tdTomato-F-positive dl1 growth cone (white arrow, shown in red). r, rostral; c, caudal; d, dorsal; v, ventral.

Movie 20. Whole-mount staining of medial floor-plate cells revealed alignment between their basal feet and dl1 growth cones in vivo. Example of Hoxa1::EGFP-F-

1021 positive medial FP cells at $\mathrm{HH} 24.5$ (shown in green) with basal feet oriented in the dorso-ventral axis (white arrows). Math1::tdTomato-F-positive dl1 growth cones were aligned with the FP basal feet (white arrow, shown in red). $r$, rostral; c, caudal; $d$, dorsal; $\mathrm{v}$, ventral.

Movie 21. Z-stack animation of the medial floor-plate area reveal protrusions of basal feet and demonstrated close interaction between dl1 axons and floor-plate basal feet in the commissure in vivo. Immunohistochemistry of $\mathrm{HH} 22$ spinal cord sections revealed that Hoxa1::EGFP-F-positive medial FP basal feet (shown in green) also contained protrusions in the commissure in vivo (white arrows). FP basal feet seemed to enwrap Math1::tdTomato-F-positive axons in the commissure (white arrowheads). Nuclei were counterstained with Hoechst (shown in blue). d, dorsal; v, ventral.

Movie 22. Time-lapse recording sequence of a dl1 commissural growth cone 
1039 (white arrows) coming from the Hoxa1::EGFP-F-positive FP basal feet (shown in green) 1040 before entering the FP. Note that at " $\mathrm{t}=240 \mathrm{~min}$ " a Z-stack animation is shown to 1041 pinpoint the close vicinity between basal feet protrusions and the growth cone (white 1042 arrows and arrowheads, respectively). Rostral is up.

Movie 23. Time-lapse recording sequence of a dl1 commissural growth cone transiently splitting in the commissure in between floor-plate basal feet. $A$ Math1::tdTomato-F-positive dl1 growth cone (shown in red, white arrowhead) growing through the FP basal feet (shown in green) and undergoing transient splitting (white arrow). Note that at "t=2 h" (splitting time point) a Z-stack animation is shown to pinpoint that FP basal feet structures (yellow arrow) were present in between both branches of the split dl1 growth cone. Rostral is up.

Movie 24. 48-hour time-lapse recording sequence showing aberrant caudal turning of dl1 commissural axons after silencing Fzd3. Math1::EGFP-F; miFzd3positive dl1 axons exiting the FP are shown in black. The turning behavior at the contralateral FP border was randomized with a substantial number of mutant dl1 axons turning caudally instead of rostrally (black arrows). Often, collective behavior was seen, that is, after a first axon turning caudally many other growth cones seemed to follow the same path and fasciculated with axons that turned in the wrong direction (black asterisk). Dashed line represents the FP exit site. Rostral is up.

Movie 25. 48-hour time-lapse recording showing growth cone stalling at the floorplate exit site after silencing Fzd3. Math1::EGFP-F; miFzd3-positive dl1 axons exiting 1063 the FP are shown in black. Black arrowhead shows a mutant dl1 axons stalling at the 1064 contralateral FP border. Some axons were also turning caudally (black arrows). Dashed 1065 line represents the FP exit site. Rostral is up. 
Movie 26. 48-hour time-lapse recording showing normal behavior of controltreated dl1 axons at floor-plate exit. Math1::EGFP-F; mi2Luc-positive dl1 axons exiting the FP are shown in black. All growth cones behaved normally at the contralateral FP border and turned rostrally (black arrowheads). Dashed line represents the FP exit site. Rostral is up.
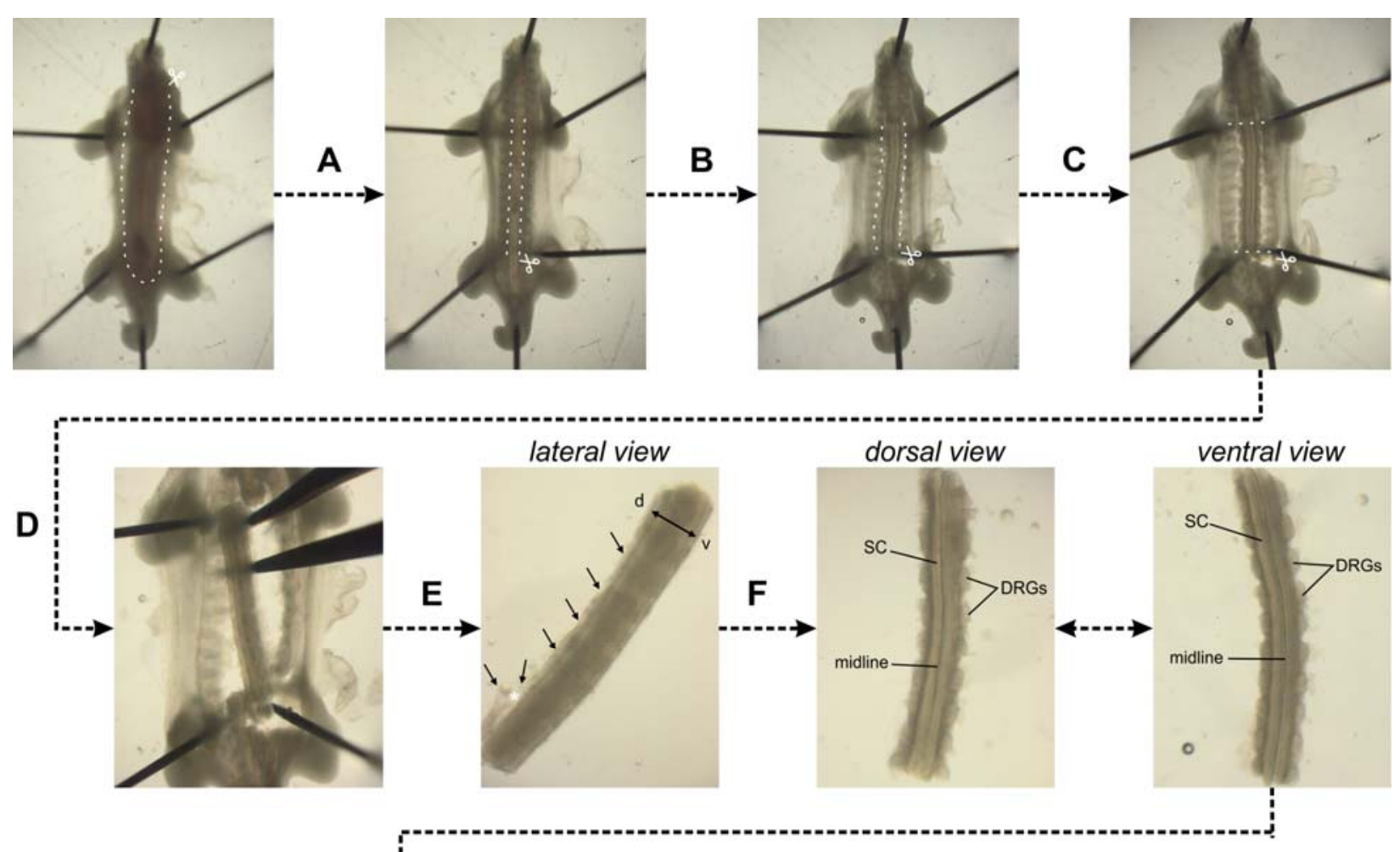

Figure S1, Dumoulin et al.

Fig. S1. Dissection of intact spinal cords from HH22 chicken embryos. 
1077 (A) HH22 embryos were pinned down with the dorsal side down in a silicon-coated Petri 1078 dish in sterile, cold PBS. Internal organs were removed by first cutting the ventral skin 1079 along the dashed lines and pinching out the organs with forceps. (B) Then, a 1080 laminectomy was performed, i.e. the ventral vertebrae were cut along the caudal-rostral 1081 axis at the level of the outer spinal cord boundaries and the stripe of bone structure was 1082 removed with forceps. (C) The visible ventral roots exiting the ventral part of the spinal 1083 cord and the peripheral processes of the dorsal root ganglia (DRG) were cut in parallel 1084 to the spinal cord without cutting off any DRG. (D) The spinal cord was then cut at the 1085 level of the wings and legs. (E) The spinal cord with attached DRG was carefully 1086 separated from the rest of the embryo with forceps. Here, special care should be given 1087 not to bend the spinal cord by stabilizing the tissue with a second forceps. (F) At this 1088 point, the dorsal skin and dermomyotome (black arrows) were removed by first inducing 1089 an opening with forceps (white asterisk) taking care not to damage the dorsal spinal 1090 cord. Then, using forceps, the dorsal skin and dermomyotome were carefully removed 1091 all along the caudal-rostral axis. After this step the dorsal spinal cord should look as 1092 clean as the ventral spinal cord with clearly visible midline and no remaining tissues 1093 attached (compare dorsal and ventral view). (G) Finally, the intact spinal cord with 1094 attached DRG could be embedded as straight as possible in a drop of low-melting 1095 agarose-medium mix with the ventral side down. White dashed lines indicate where cuts 1096 with small spring scissors should be made. SC, spinal cord; DRG, dorsal root ganglion. 
bioRxiv preprint doi: https://doi.org/10.1101/2020.07.20.211995; this version posted July 20, 2020. The copyright holder for this preprint (which was not certified by peer review) is the author/funder, who has granted bioRxiv a license to display the preprint in perpetuity. It is made available under aCC-BY-NC-ND 4.0 International license.

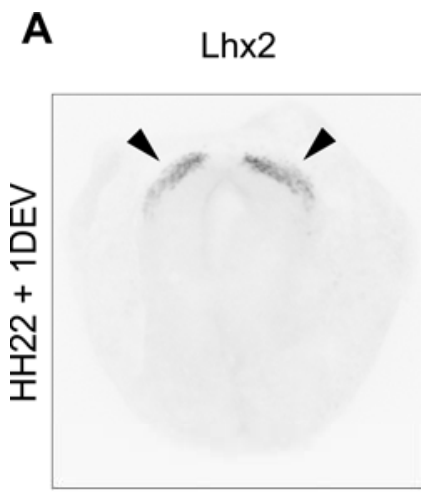

B

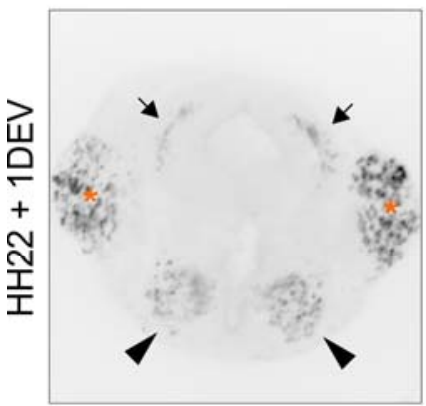

C

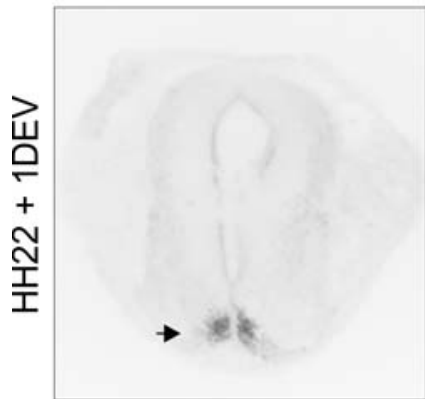

D

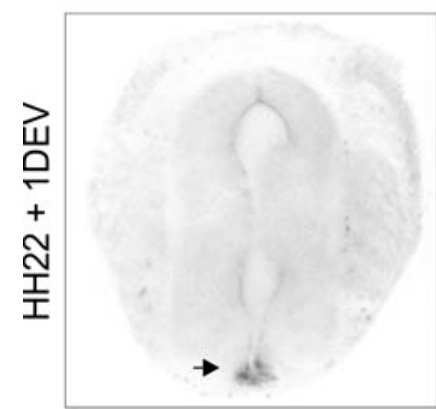

Math1::tdTomato-F

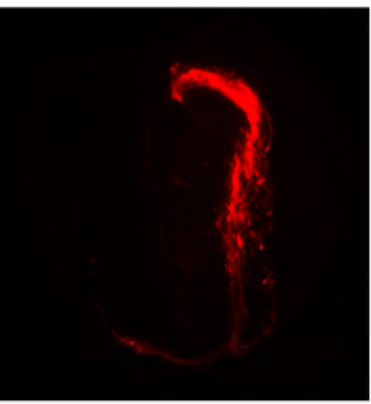

Math1::tdTomato-F

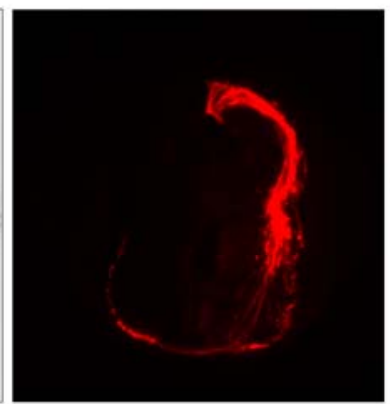

Math1::tdTomato-F

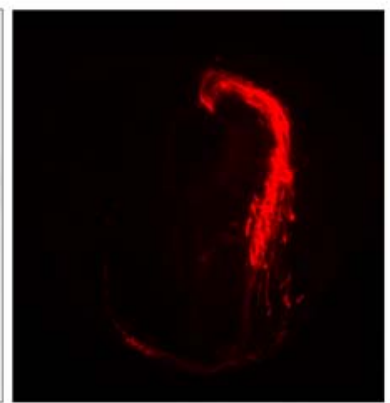

Math1::tdTomato-F

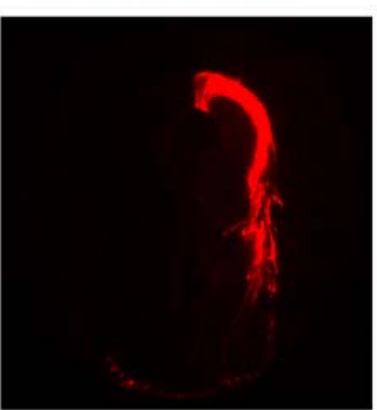

Math1::tdTomato-F Lhx2

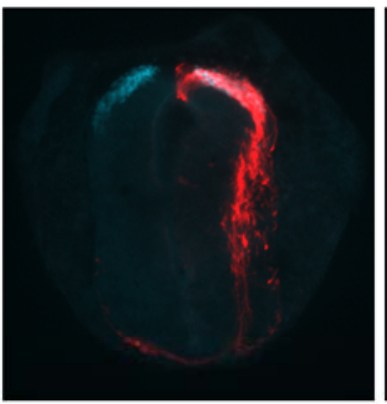

Math1::tdTomato-F Islet-1

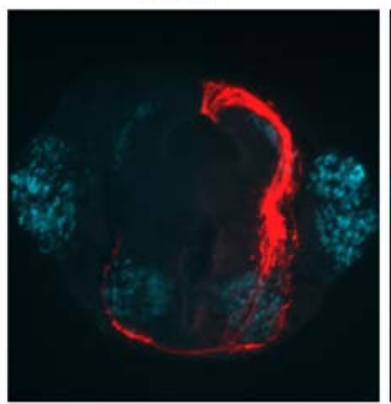

Math1::tdTomato-F $\mathrm{Nk} \times 2.2$

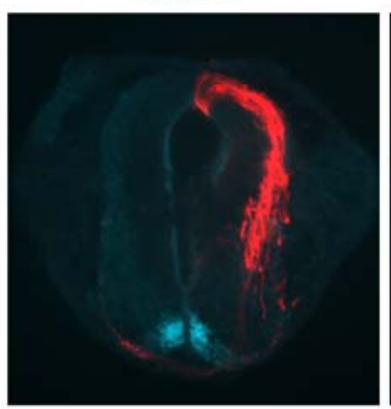

Math1::tdTomato-F $\mathrm{Hnf3 \beta}$

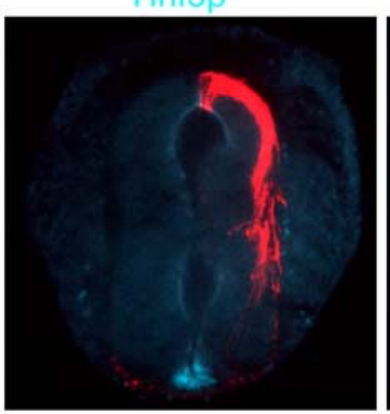

Math1::tdTomato-F Hnf $3 \beta$ Hoechst

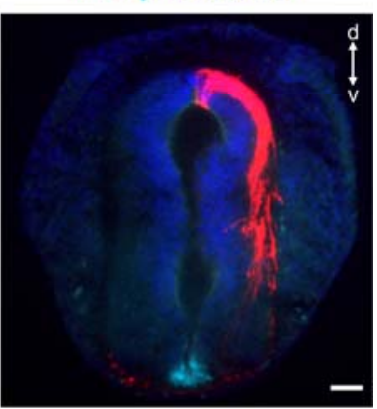


1101 Fig. S2. Patterning of cultured intact spinal cords was conserved after one day ex

1102 vivo.

1103 After intact HH22 spinal cords were cultured and imaged for 1 day ex vivo, they were 1104 fixed and transverse cryosections were immunostained for different dorsal and ventral 1105 patterning markers, RFP (Math1-positive dl1 neurons) and counterstained with Hoechst.

1106 (A) The dl1 interneuron marker Lhx2 confirmed that these neurons were still localized in 1107 the most dorsal part of the spinal cord, as expected (black arrowheads). (B) Islet-1 was 1108 used as a marker for DRG neurons (orange asterisks), dl3 interneurons (black arrows) 1109 and motoneurons (black arrowheads). All of them maintained the appropriate position: 1110 clustered DRG neurons adjacent to the spinal cord; dl3 interneurons localized ventrally 1111 of dl1 interneurons; motoneurons on both sides of the ventral spinal cord. (C) Nkx2.2 1112 staining was used to reveal the ventral population of V3 progenitors that are just next to 1113 the FP and form the typical inverted V-shape (black arrow). (D) Finally, FP cells forming 1114 the intermediate target for dl1 axons were visualized with Hnf3 $\beta$ staining. They were 1115 localized at the ventral midline of the spinal cord as expected (black arrow). DEV, day 1116 ex vivo; $d$, dorsal; v, ventral. Scale bars: $50 \mu \mathrm{m}$. 


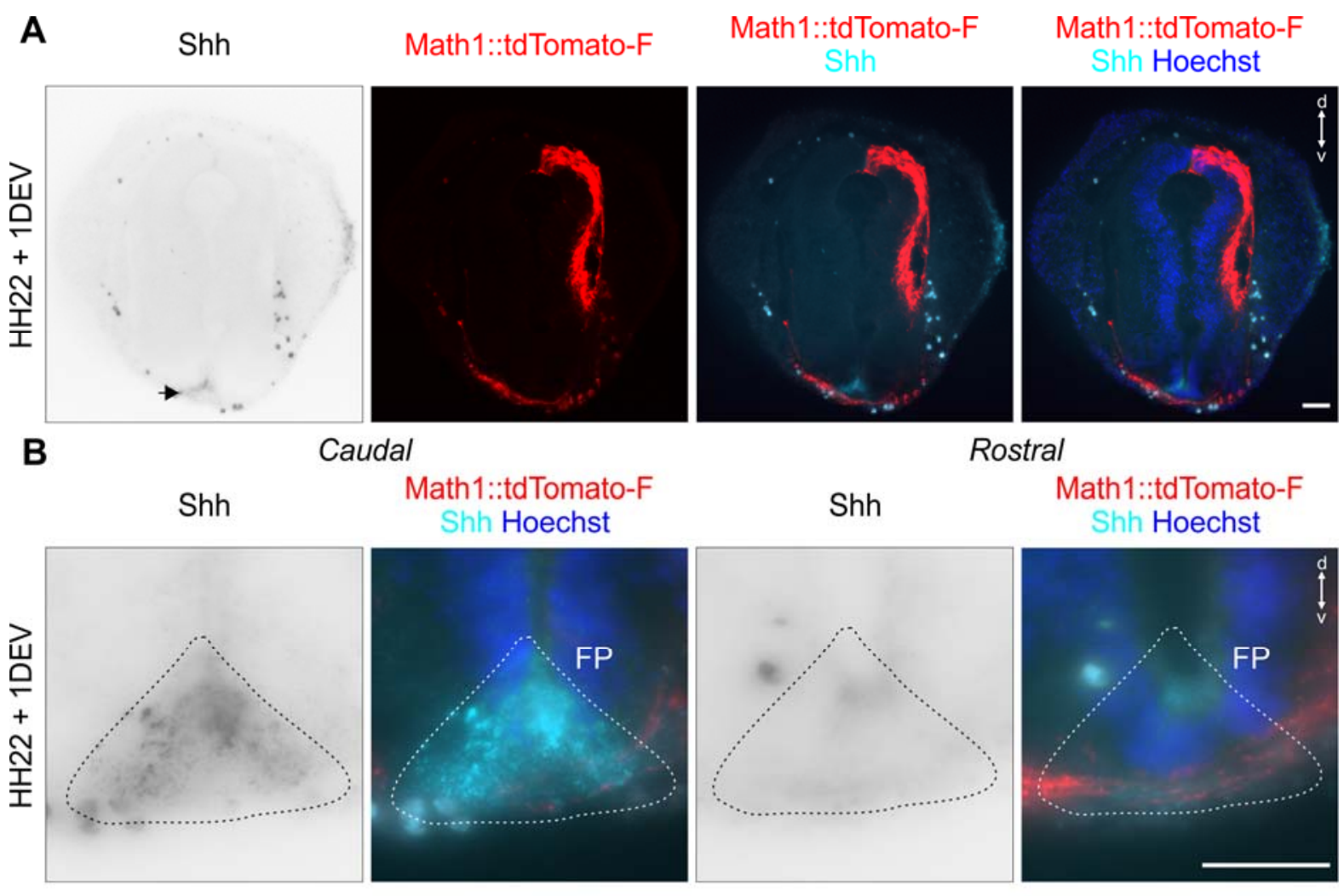

1119 Fig. S3. A Shh gradient is still present in a cultured intact spinal cord after one 1120 day ex vivo.

1121 Intact HH22 spinal cords were cultured and imaged for 1 day ex vivo before fixation.

1122 Transverse cryosections were immunostained with antibodies against Shh (5E1 clone), 1123 RFP (Math1-positive dl1 neurons) and counterstained with Hoechst. (A) Shh was still 1124 expressed in the FP after one day ex vivo (black arrow). (B) Moreover, in agreement 1125 with previous descriptions in vivo, Shh was expressed in a decreasing caudal-to-rostral 1126 gradient. d, dorsal; v, ventral. Scale bars: $50 \mu \mathrm{m}$. 
A HH22 + Oh ex vivo HH22 +12h ex vivo HH22 +24h ex vivo

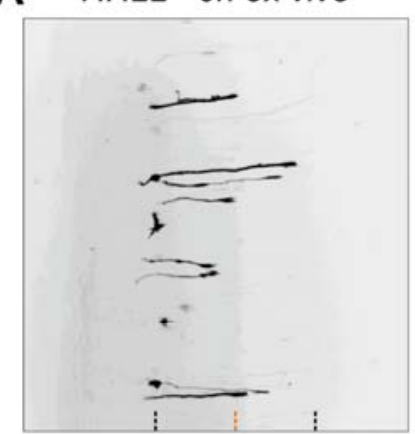

C Math1::tdTomato-F

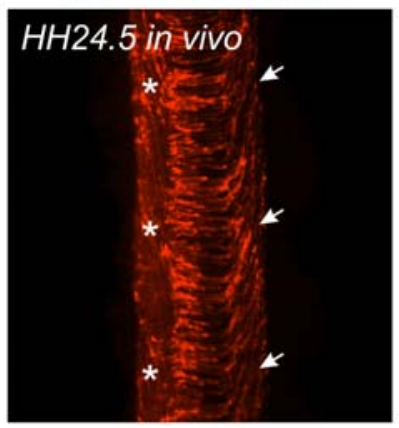

1128

1129

1130

1131

1132

1133

1134

1135

1136

1137

1138

1139

1140

1141

1142

1143

1144

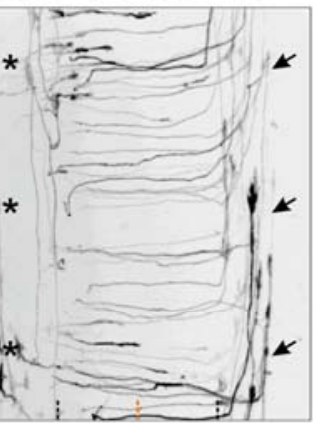

B-actin::EGFP-F

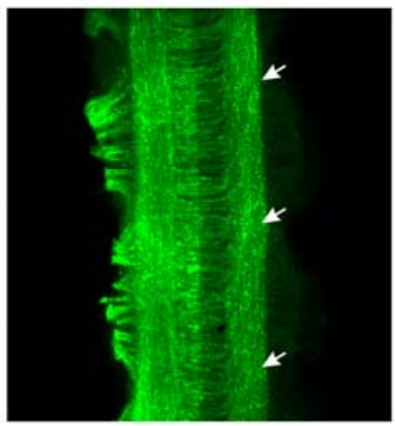

Hoechst

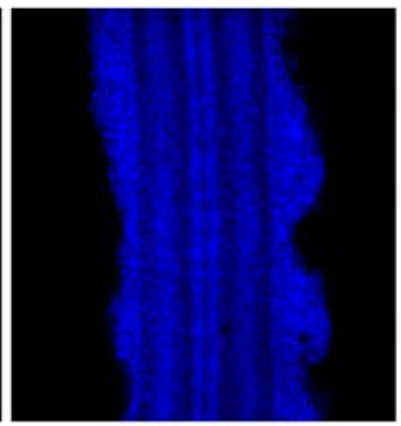

Fig. S4. Development of Math1-positive axonal tracts ex vivo and in vivo. asterisks). Scale bars: $50 \mu \mathrm{m}$ (A and B) and $100 \mu \mathrm{m}(C)$.
B HH25 in vivo

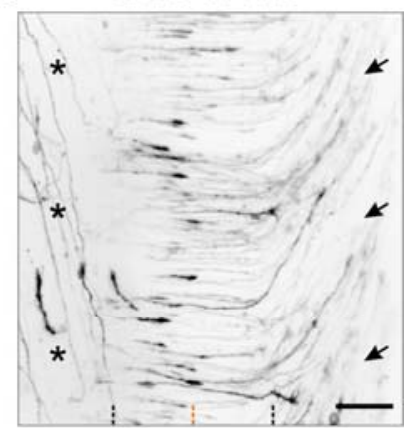

Brightfield

Figure S4, Dumoulin et al.

(A) Sequence of 3 images showing dl1 axons crossing the FP after 0,12 and $24 \mathrm{~h}$ of culture. After turning rostrally, post-crossing axons started to form the contralateral ventral funiculus (black arrows). After around 12h in culture, a Math1-positive ipsilateral population could be clearly seen in the ipsilateral ventral funiculus (black asterisks). (B) Intact spinal cords dissected at $\mathrm{HH} 25$, fixed and mounted similarly to the ex vivo culture were imaged the same way. This revealed identical dl1 axonal tracts compared to those seen after $24 \mathrm{~h}$ of culture of intact spinal cords dissected at $\mathrm{HH} 22$, with post-crossing axons forming the contralateral ventral funiculus (black arrows) and ipsilateral axons turning in the ipsilateral ventral funiculus (black asterisks). Black and orange dashed lines represent FP boundaries and midline, respectively. (C) Low magnification overview of an intact HH24.5 spinal cord, fixed, stained for RFP (Math1-positive neurons) and GFP ( $\beta$-actin transfected cells) and counterstained with Hoechst showing the contralateral ventral funiculus containing post-crossing dl1 axons (white arrows) and the ipsilateral ventral funiculus containing a population of ipsilateral axons (white 
A

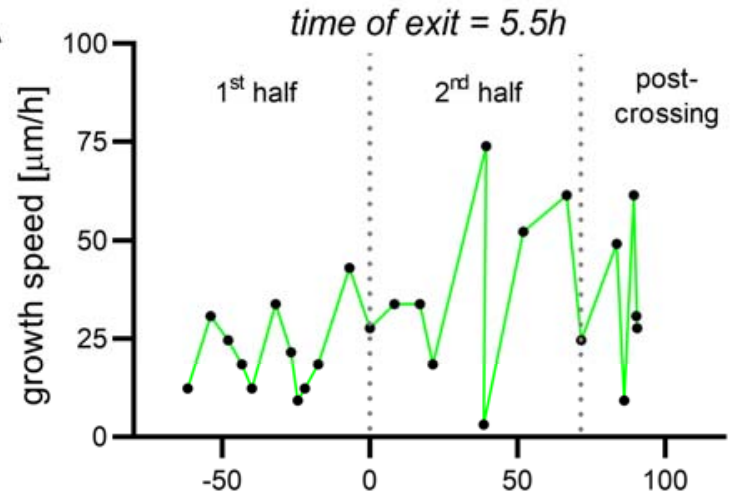

position versus midline $[\mu \mathrm{m}]$

C

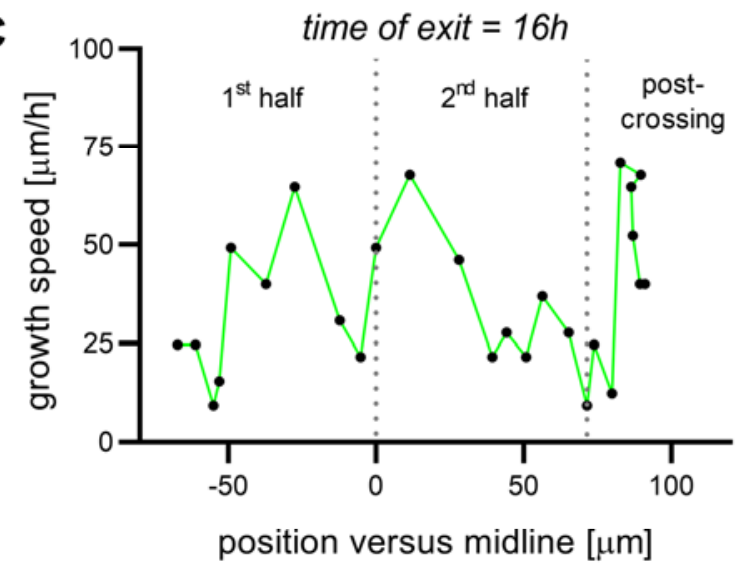

E

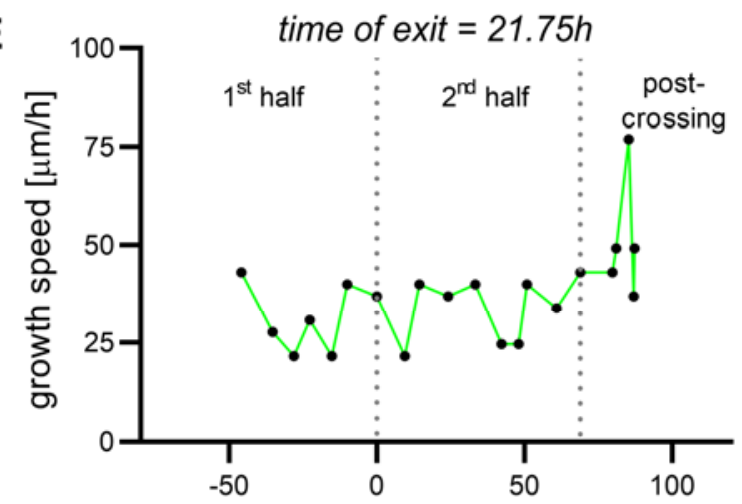

position versus midline $[\mu \mathrm{m}]$
B

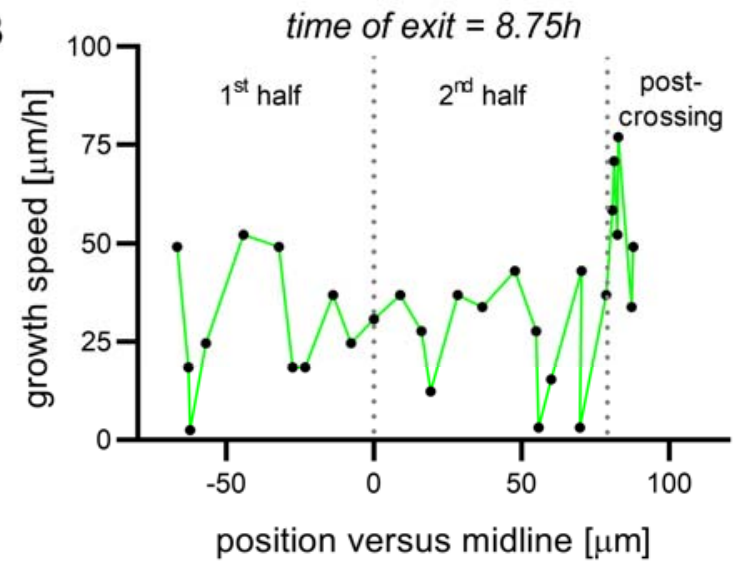

D

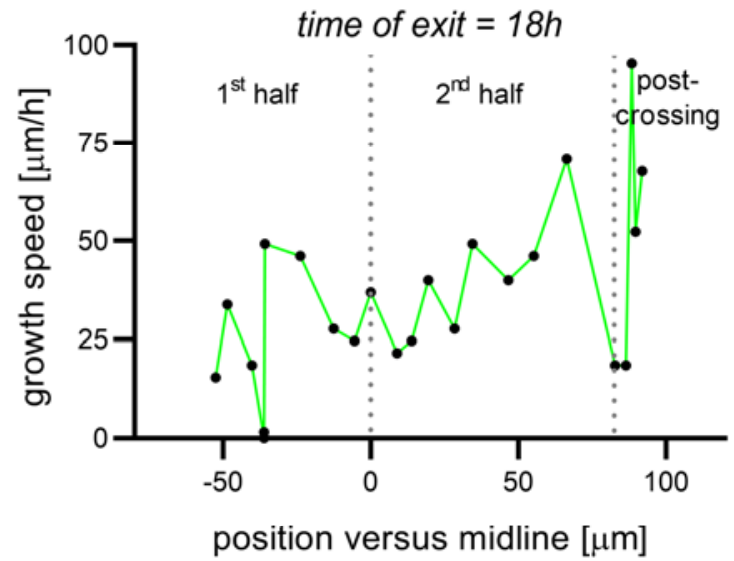

$\mathbf{F}$

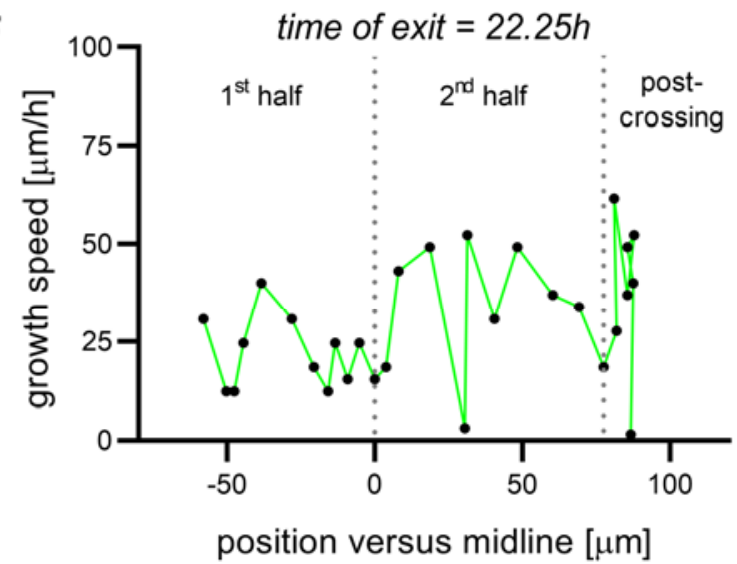

Figure S5, Dumoulin et al. 
1150 Fig. S5. Virtual tracing of axons exiting the FP at different time points.

1151 Examples of instantaneous growth speed of axons exiting the FP after 5.5h (A), 8.75h

1152 (B), 16h (C), 18h (D), 21.75h (E) or 22.25h (F) of culture that could be extracted and 1153 plotted against the position of the growth cone in the FP. Dotted lines represent the time 1154 at which the axon crossed the midline or exited the FP. All axons were growing with 1155 pulses of acceleration and deceleration. There was no difference between early or late 1156 crossing axons. 


\begin{tabular}{|c|c|c|c|c|}
\hline Fig. & Name & Mean & Standard deviation & \\
\hline \multirow{5}{*}{ Fig.3B } & Ent-Mid & 2.81 & 0.96 & $\mathrm{n}($ axons $)=298, \mathrm{~N}($ embryos $)=7$ \\
\hline & Mid-Ex & 2.74 & 0.95 & $\mathrm{n}($ axons $)=298, \mathrm{~N}($ embryos $)=7$ \\
\hline & Ent-Ex & 5.55 & 1.39 & $\mathrm{n}($ axons $)=298, \mathrm{~N}($ embryos $)=7$ \\
\hline & Ex-Turn & 1.39 & 1.03 & $\mathrm{n}($ axons $)=298, \mathrm{~N}($ embryos $)=7$ \\
\hline & Ent-Turn & 6.94 & 1.79 & $\mathrm{n}($ axons $)=298, \mathrm{~N}($ embryos $)=7$ \\
\hline \multirow{4}{*}{ Fig.4B } & 1st half & 46.1 & 12.2 & $\mathrm{n}$ (growth cones $)=127, \mathrm{~N}($ embryos $)=7$ \\
\hline & 2nd half & 44.2 & 11 & $\mathrm{n}$ (growth cones $)=127, \mathrm{~N}($ embryos $)=7$ \\
\hline & exit & 105.5 & 25 & $\mathrm{n}$ (growth cones $)=127, \mathrm{~N}($ embryos $)=7$ \\
\hline & after turn & 67.8 & 17.6 & $\mathrm{n}$ (growth cones $)=127, \mathrm{~N}$ (embryos $)=7$ \\
\hline \multirow{4}{*}{ Fig.4C } & 1st half & 41 & 12.6 & $\mathrm{n}$ (growth cones $)=285, \mathrm{~N}($ embryos $)=8$ \\
\hline & 2nd half & 41.8 & 11.5 & $\mathrm{n}$ (growth cones $)=153, \mathrm{~N}($ embryos $)=8$ \\
\hline & exit & 116.9 & 25.7 & $\mathrm{n}($ growth cones $)=68, \mathrm{~N}($ embryos $)=8$ \\
\hline & after turn & 76.5 & 18.9 & $\mathrm{n}$ (growth cones $)=102, \mathrm{~N}($ embryos $)=8$ \\
\hline
\end{tabular}

